\title{
Geostrophic Turbulence in the Frequency-Wavenumber Domain: Eddy-Driven Low-Frequency Variability*
}

\author{
BriAn K. ARBIC, ${ }^{+, @ @ ~ M A L T E ~ M U ̈ L L E R, ~}$, ,@,$\& \&$ JAMES G. RICHMAN, ${ }^{@}$ JAY F. SHRIVER, ${ }^{@}$ \\ Andrew J. Morten, ${ }^{\&}$ Robert B. SCOTT, ${ }^{* *}$ GuIllaume SÉrAzIn, ${ }^{++}$AND THIERRY PendufF ${ }^{\#}$ \\ ${ }^{+}$Department of Earth and Environmental Sciences, University of Michigan, Ann Arbor, Michigan \\ ${ }^{\#}$ School of Earth and Ocean Sciences, University of Victoria, Victoria, British Columbia \\ @ Oceanography Division, Naval Research Laboratory, Stennis Space Center, Mississippi \\ ${ }^{\&}$ Department of Physics, University of Michigan, Ann Arbor, Michigan \\ ** Institute for Geophysics, Jackson School of Geosciences, The University of Texas at Austin, Austin, Texas, \\ and Departement de Physique et LPO, Université de Bretagne Occidentale, CNRS, Brest, France \\ ${ }^{++}$Laboratoire de Glaciologie et Géophysique de l'Environnement, CNRS, and Université Grenoble Alpes, Grenoble, \\ and Centre Européen de Recherche et de Formation Avancée en Calcul Scientifique, Toulouse, France \\ \#\# Laboratoire de Glaciologie et Géophysique de l'Environnement, CNRS, and Université \\ Grenoble Alpes, Grenoble, France
}

(Manuscript received 7 March 2013, in final form 30 March 2014)

\begin{abstract}
Motivated by the potential of oceanic mesoscale eddies to drive intrinsic low-frequency variability, this paper examines geostrophic turbulence in the frequency-wavenumber domain. Frequency-wavenumber spectra, spectral fluxes, and spectral transfers are computed from an idealized two-layer quasigeostrophic (QG) turbulence model, a realistic high-resolution global ocean general circulation model, and gridded satellite altimeter products. In the idealized QG model, energy in low wavenumbers, arising from nonlinear interactions via the well-known inverse cascade, is associated with energy in low frequencies and vice versa, although not in a simple way. The range of frequencies that are highly energized and engaged in nonlinear transfer is much greater than the range of highly energized and engaged wavenumbers. Lowfrequency, low-wavenumber energy is maintained primarily by nonlinearities in the QG model, with forcing and friction playing important but secondary roles. In the high-resolution ocean model, nonlinearities also generally drive kinetic energy to low frequencies as well as to low wavenumbers. Implications for the maintenance of low-frequency oceanic variability are discussed. The cascade of surface kinetic energy to low frequencies that predominates in idealized and realistic models is seen in some regions of the gridded altimeter product, but not in others. Exercises conducted with the general circulation model suggest that the spatial and temporal filtering inherent in the construction of gridded satellite altimeter maps may contribute to the discrepancies between the direction of the frequency cascade in models versus gridded altimeter maps seen in some regions. Of course, another potential reason for the discrepancy is missing physics in the models utilized here.
\end{abstract}

\footnotetext{
* Naval Research Laboratory Contribution Number NRL/JA/ 7320-12-1515 and University of Texas Institute for Geophysics Contribution Number 2638.

@@ These authors contributed equally to this manuscript.

\&\& Current affiliation: Research and Development Department, Norwegian Meteorological Institute, Oslo, Norway.
}

Corresponding author address: Dr. Brian K. Arbic, Department of Earth and Environmental Sciences, University of Michigan, 1100 North University Avenue, Ann Arbor, MI 48109-1005.

E-mail: arbic@umich.edu

\section{Introduction}

Geostrophic turbulence and its progenitor twodimensional turbulence serve as valuable paradigms for atmospheric and oceanic flows. This paper addresses the relative contributions of forcing versus intrinsic nonlinearities in the maintenance of low-frequency variability in oceanic geostrophic turbulence. Our main diagnostics are spectral fluxes and spectral transfers-long utilized in wavenumber space analyses of geostrophic turbulencecomputed here in frequency-wavenumber space. 
The role of forcing versus intrinsic nonlinear processes in the maintenance of low-frequency variability is an important topic in climate dynamics. The climate system exhibits variability over a vast range of time scales. Huybers and Curry (2006) show, for instance, that the frequency spectrum of surface temperature from monthly out to 100000 -yr time scales displays power at all frequencies. Some low-frequency variability is due to forcing. Hasselmann (1976) shows that an idealized linear ocean with long response times will redden the spectrum of a white noise forcing. Similarly, Griffies and Tziperman (1995) show that high-frequency stochastic atmospheric forcing excites low frequency modes in the ocean. A number of studies implicate intrinsic nonlinear processes, in both the atmosphere and ocean, as another important contributor to low-frequency variability (e.g., Holland 1978; Sheng and Hayashi 1990a,b; Spall 1996; Berloff and McWilliams 1999; Dijkstra and Molemaker 1999; Dewar 2001). Recently, Penduff et al. (2011) demonstrate that interannual variance of sea surface height in ocean general circulation models driven by atmospheric forcing fields that lack interannual variability is comparable to the variance in ocean models driven by interannually varying forcing fields. However, these variances are comparable if and only if the ocean models are turbulent [or, using terminology borrowed from Hecht and Hasumi (2008), "eddying"'. Penduff et al. (2011) and related studies (e.g., Taguchi et al. 2010) therefore suggest a role for mesoscale eddies in the maintenance of oceanic low-frequency variability.

As pointed out by Ferrari and Wunsch (2010), the theory of quasi-two-dimensional turbulence is less well developed in the frequency domain than in the wavenumber domain. Numerous studies (e.g., Fjortoft 1953; Batchelor 1953; Kraichnan 1967, 1971; Charney 1971; Vallis 2006) argue that nonlinearities in two-dimensional and quasigeostrophic (QG) turbulence drive an inverse cascade of energy toward larger length scales (lower wavenumbers). Scott and Wang (2005) document an inverse cascade in the surface ocean, strongly suggesting that the ocean is in a state of geostrophic turbulence. Arbic et al. (2012b, hereinafter ASFMRS) demonstrate that nonlinearities in an idealized, two-layer, $f$-plane, $\mathrm{QG}$ turbulence model forced by an imposed, baroclinically unstable, mean flow (e.g., Salmon 1978, 1980; Haidvogel and Held 1980; Larichev and Held 1995) drive energy toward longer time scales (lower frequencies), alongside the well-known inverse cascade to lower wavenumbers. ASFMRS also show that nonlinearities play a larger role than forcing and damping in the maintenance of lowfrequency variance, at least in baroclinically unstable $f$-plane $\mathrm{QG}$ turbulence. The frequency domain spectral transfers and fluxes used in ASFMRS provide a new tool to measure the relative importance of nonlinear intrinsic processes versus forcing in the maintenance of lowfrequency oceanic variability. ASFMRS show that nonlinearities also generally drive energy to longer time scales in realistic eddying ocean general circulation models. We note that the term "forcing" can mean different things in different settings. In our idealized QG model the forcing is an imposed, horizontally homogeneous, temporally fixed, baroclinically unstable mean flow. In the realistic models used by Penduff et al. (2011) and Taguchi et al. (2010), forcing denotes the atmospheric fields, which vary in complex ways in both space and time.

Here we extend the frequency domain analysis of ASFMRS to the frequency-wavenumber domain. Frequency-wavenumber analysis allows inspection of the behaviors at low frequencies versus low wavenumbers, a determination of which wavenumbers are primarily responsible for nonlinearity at low frequencies, and a determination of which frequencies are primarily responsible for nonlinearity at low wavenumbers. Frequency-wavenumber spectra provide a test of the Taylor (1938) hypothesis relating frequency spectra to wavenumber spectra. Finally, as will be seen, the analysis of the frequency-wavenumber spectral fluxes provides a constraint on the values of the frequency domain spectral fluxes at the lowest frequencies, which in turn allows for a closer comparison of the low-frequency fluxes in QG versus realistic models than was done in ASFMRS. Previous studies of oceanic frequencywavenumber spectra include Wunsch and Stammer (1995), Chelton and Schlax (1996), Farrar (2008), Wunsch (2009, 2010), Early et al. (2011), and Wortham (2013), among others. A comprehensive discussion of frequency-wavenumber spectra computed from models, satellite altimetry data, and in situ data can be found in Wortham (2013). Hayashi and Golder (1977) and Hayashi (1982) discuss the "space-time" (wavenumberfrequency) spectra of realistic atmospheric models and datasets. We are not aware of any previous studies examining frequency-wavenumber domain spectral fluxes and transfers in idealized QG turbulence models, or in an oceanic context, as we do here. Note that our focus here is on isotropic wavenumber-frequency spectral diagnostics. In a companion paper, we analyze spectral diagnostics in the anisotropic wavenumber-frequency domain to better understand the role of nonlinearities in westward eddy propagation.

We examine output from the same two-layer QG turbulence model utilized in ASFMRS. However, the realistic eddying ocean model utilized here is the Hybrid Coordinate Ocean Model (HYCOM; Chassignet et al. 2007), in place of the Naval Research Laboratory (NRL) Layered Ocean Model (NLOM; Hurlburt and 
Thompson 1980; Shriver et al. 2007) used in ASFMRS. HYCOM has more layers in the vertical direction than does NLOM and a number of other more realistic features.

As in ASFMRS and Arbic et al. (2013), we will also compute spectral diagnostics from a satellite altimeter product. The Archiving, Validation, and Interpretation of Satellite Oceanographic (AVISO) gridded satellite altimeter product (Le Traon et al. 1998; Ducet et al. 2000) we use is constructed through interpolation, in space and time, of along-track sea surface height (SSH) data from two altimeters onto a $1 / 3^{\circ}$ Mercator grid at regular 7-day intervals. Because interpolation smooths the along-track data, the spatial feature resolution of AVISO maps is coarser than the $\sim 6-\mathrm{km}$ resolution along track and is closer to the $\sim 300-\mathrm{km}$ cross-track spacing of the Ocean Topography Experiment (TOPEX)/Poseidon and Jason altimeters (Chelton et al. 2011). Wortham (2013) finds that the AVISO product has significantly lower energy than raw, along-track altimetry data for wavelengths shorter than about $225 \mathrm{~km}$ and periods shorter than about 55 days. We investigate the impact of the smoothing inherent in the construction of AVISO products through comparison of spectral fluxes computed from filtered versions of the realistic model output with fluxes computed from unadulterated output.

This paper is organized as follows: In section 2, we describe the models and altimeter product. In section 3 , we discuss the theory of frequency-wavenumber domain spectral fluxes and transfers. Section 4 presents the analysis technique, and section 5 presents the results. A summary and discussion is given in section 6 .

\section{Description of models and gridded altimeter product}

\section{a. Idealized quasigeostrophic turbulence model}

For simplicity, we utilize a highly idealized two-layer QG turbulence model on an $f$-plane with a flat bottom, forced by an imposed, horizontally homogeneous, vertically sheared mean flow that roughly represents oceanic gyres. The model is pseudospectral and doubly periodic, with $256 \times 256$ grid points, where each side has dimensional length $20 \pi L_{d}$. Flow begins with a randomly generated initial condition, and the model achieves statistical equilibrium when eddy energy extraction from the baroclinically unstable mean flow is balanced by energy dissipation. Despite the numerous simplifications, the frequency/isotropic wavenumber behaviors in the $\mathrm{QG}$ model compare well in many respects with the behaviors in the realistic ocean model and in gridded satellite altimeter maps.

We follow the format of Flierl (1978) in our governing equations:

$$
\begin{aligned}
& \frac{\partial q_{1}}{\partial t}+\Gamma_{1}+J\left(\psi_{1}, q_{1}\right)=\text { ssd, } \quad \text { and } \\
& \frac{\partial q_{2}}{\partial t}+\Gamma_{2}+J\left(\psi_{2}, q_{2}\right)=-R_{2} \nabla^{2} \psi_{2}+\text { ssd }
\end{aligned}
$$

where ssd is small-scale dissipation. Here $t$ is time, $q$ is the perturbation potential vorticity, and subscripts 1 and 2 , respectively, denote upper and lower layers. Perturbation streamfunctions $\psi_{1}$ and $\psi_{2}$ are related to $q$ by

$q_{1}=\nabla^{2} \psi_{1}+\frac{\left(\psi_{2}-\psi_{1}\right)}{(1+\delta) L_{d}^{2}}, \quad q_{2}=\nabla^{2} \psi_{2}+\frac{\delta\left(\psi_{1}-\psi_{2}\right)}{(1+\delta) L_{d}^{2}}$,

where $\delta=H_{1} / H_{2}$, the ratio of upper- to lower-layer depths, and the first baroclinic mode deformation radius $L_{d}$ is defined by $\left[g^{\prime} H_{1} H_{2} / f_{0}^{2}\left(H_{1}+H_{2}\right)\right]^{1 / 2}$, where $g^{\prime}$ is reduced gravity and $f_{0}$ is the Coriolis parameter. The zonal (east-west) and meridional (north-south) spatial coordinates are $x$ and $y$, respectively. The Jacobian of two fields $A$ and $B$ is given by $J(A, B)=\partial A$ / $\partial x \partial B / \partial y-\partial A / \partial y \partial B / \partial x$. The mean flow forcing terms are

$$
\begin{aligned}
& \Gamma_{1}=\overline{u_{1}} \frac{\partial q_{1}}{\partial x}+\frac{\partial \overline{q_{1}}}{\partial y} \frac{\partial \psi_{1}}{\partial x}, \text { and } \\
& \Gamma_{2}=\overline{u_{2}} \frac{\partial q_{2}}{\partial x}+\frac{\partial \overline{q_{2}}}{\partial y} \frac{\partial \psi_{2}}{\partial x},
\end{aligned}
$$

where imposed time-mean quantities are designated with overbars. The zonal and meridional velocities are denoted by $u$ and $v$, respectively. On the $f$-plane, the direction of the mean flow is arbitrary; we take it to be zonal. The imposed time-mean potential vorticity gradients are

$$
\frac{\partial \overline{q_{1}}}{\partial y}=\frac{\left(\overline{u_{1}}-\overline{u_{2}}\right)}{(1+\delta) L_{d}^{2}}, \quad \frac{\partial \overline{q_{2}}}{\partial y}=\frac{\delta\left(\overline{u_{2}}-\overline{u_{1}}\right)}{(1+\delta) L_{d}^{2}} .
$$

The bottom Ekman drag $R_{2}$ is related to the bottom boundary layer thickness $d_{\text {Ekman }}=\sqrt{A / f_{0}}$ by

$$
R_{2}=\frac{f_{0} d_{\text {Ekman }}}{2 H_{2}}
$$

where $A$ is the vertical eddy viscosity. We use an exponential cutoff filter in wavenumber space (Canuto et al. 1988; LaCasce 1996) for ssd:

filter $=\exp \left[-\alpha\left(\sqrt{k^{2}+l^{2}}-k_{0}\right)^{M}\right]$ when $\sqrt{k^{2}+l^{2}}>k_{0}$ filter $=1.0$ when $\sqrt{k^{2}+l^{2}} \leq k_{0}$, 
where $k$ and $l$ are zonal and meridional wavenumbers, respectively, and $k_{0}$ is a cutoff wavenumber. As in LaCasce (1996), which can be consulted for more details, we take $\alpha=18.4, M=4$, and $k_{0}=0.65 k_{N}$, where $k_{N}$ corresponds to the Nyquist scale. At every time step the filter is applied to $\widehat{q_{1}}$ and $\widehat{q_{2}}$, the Fourier transforms of $q_{1}$ and $q_{2}$.

Our QG equations are governed by two nondimensional parameters. We set the stratification parameter $\delta$ to 0.2 , which yields the strength of the first baroclinic mode self-interaction in the two-layer model consistent with that obtained from typical midlatitude stratification profiles (Flierl 1978; Fu and Flierl 1980). The nondimensional linear bottom Ekman friction strength, $F_{L}=R_{2} L_{d} /\left(\bar{u}_{1}-\bar{u}_{2}\right)$, is set to 0.4 , which yields amplitudes, horizontal scales, and vertical structure of eddy kinetic energy (EKE) that agree reasonably well with midlatitude observations (Arbic and Flierl 2004; Arbic et al. 2013). Figure 1 displays representative snapshots of $\psi_{1}$ and $q_{1}$ for the QG model. The flow field consists of densely packed eddies in qualitative similarity to a typical snapshot of midocean SSH. Isolated coherent vortices (e.g., McWilliams 1984) can be seen in the $\psi_{1}$ and especially $q_{1}$ fields. The spectral diagnostics are calculated from 5400 snapshots, each spaced one unit of nondimensional time $L_{d} /\left(\bar{u}_{1}-\bar{u}_{2}\right)$ apart. The correlation time (as measured by the zero crossing in the autocorrelation of $\psi_{1}$ ) is about 16.5 units of nondimensional time, suggesting that our sampling rate is adequate.

\section{b. Global eddying general circulation model}

For details on global HYCOM the reader is directed to Metzger et al. (2010) and references therein. A brief summary is given here. The HYCOM simulation used in this paper is nonassimilative, has 32 layers in the vertical direction, and has a horizontal resolution of $1 / 12.5^{\circ}$ at the equator. The simulation was run interannually over the period July 2003-December 2010 using 3-hourly Fleet Numerical Oceanography Center Navy Operational Global Atmospheric Prediction System (FNMOC NOGAPS; Rosmond et al. 2002) atmospheric forcing with wind speeds scaled to be consistent with Quick Scatterometer (QuikSCAT; e.g., Liu and Xie 2006) observations. Comparison to surface drifters and deep current meters demonstrates that HYCOM generates a realistic circulation, albeit somewhat deficient in EKE, being only $79 \%$ and $80 \%$ of the observations at the surface and abyssal ocean (Thoppil et al. 2011). Because we are limited by the substantial computational resources needed to run global eddying HYCOM and to store its output, we have only 5 yr (2005-09) of HYCOM SSH output for our analysis. Storage concerns preclude us from examining depth-integrated energy budgets in (a) $\psi_{1}$

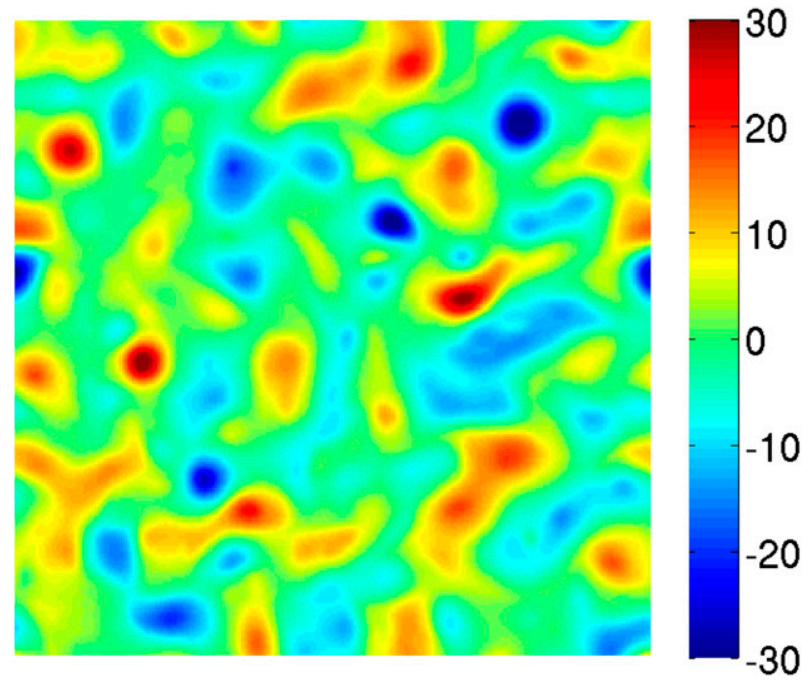

(b) $q_{1}$

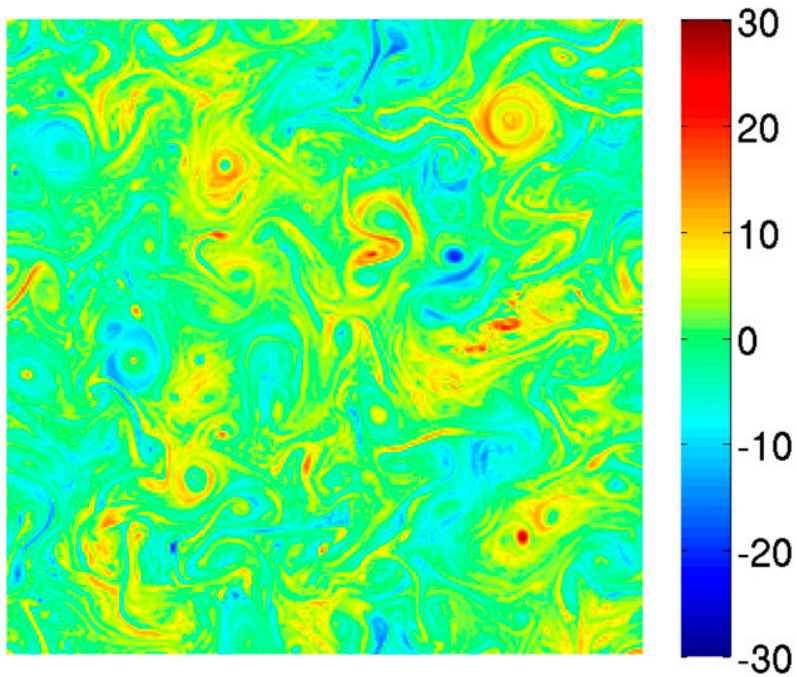

FIG. 1. Representative snapshots of (a) upper-layer streamfunction $\psi_{1}$ [normalized by $\left(\bar{u}_{1}-\bar{u}_{2}\right) L_{d}$ ] and (b) upper-layer potential vorticity $q_{1}$ [normalized by $\left(\bar{u}_{1}-\bar{u}_{2}\right) / L_{d}$ ] in idealized two-layer QG simulation. The domain is doubly periodic and $20 \pi L_{d}$ on a side, where $L_{d}$ is the first baroclinic mode Rossby deformation radius.

HYCOM, as we do in our QG analysis. Instead we focus on the spectra, spectral fluxes, and spectral transfers of geostrophic kinetic energy in HYCOM's sea surface. We choose to analyze a HYCOM simulation forced by the astronomical tidal potential as well as atmospheric fields (Arbic et al. 2010, 2012a; Shriver et al. 2012; Richman et al. 2012) so that ongoing frequency-wavenumber analyses of tidal and near-inertial flows can be easily compared to the analyses of geostrophic flows performed here. A 3-day low-pass filter is employed to remove highfrequency motions from the hourly HYCOM SSH fields, 

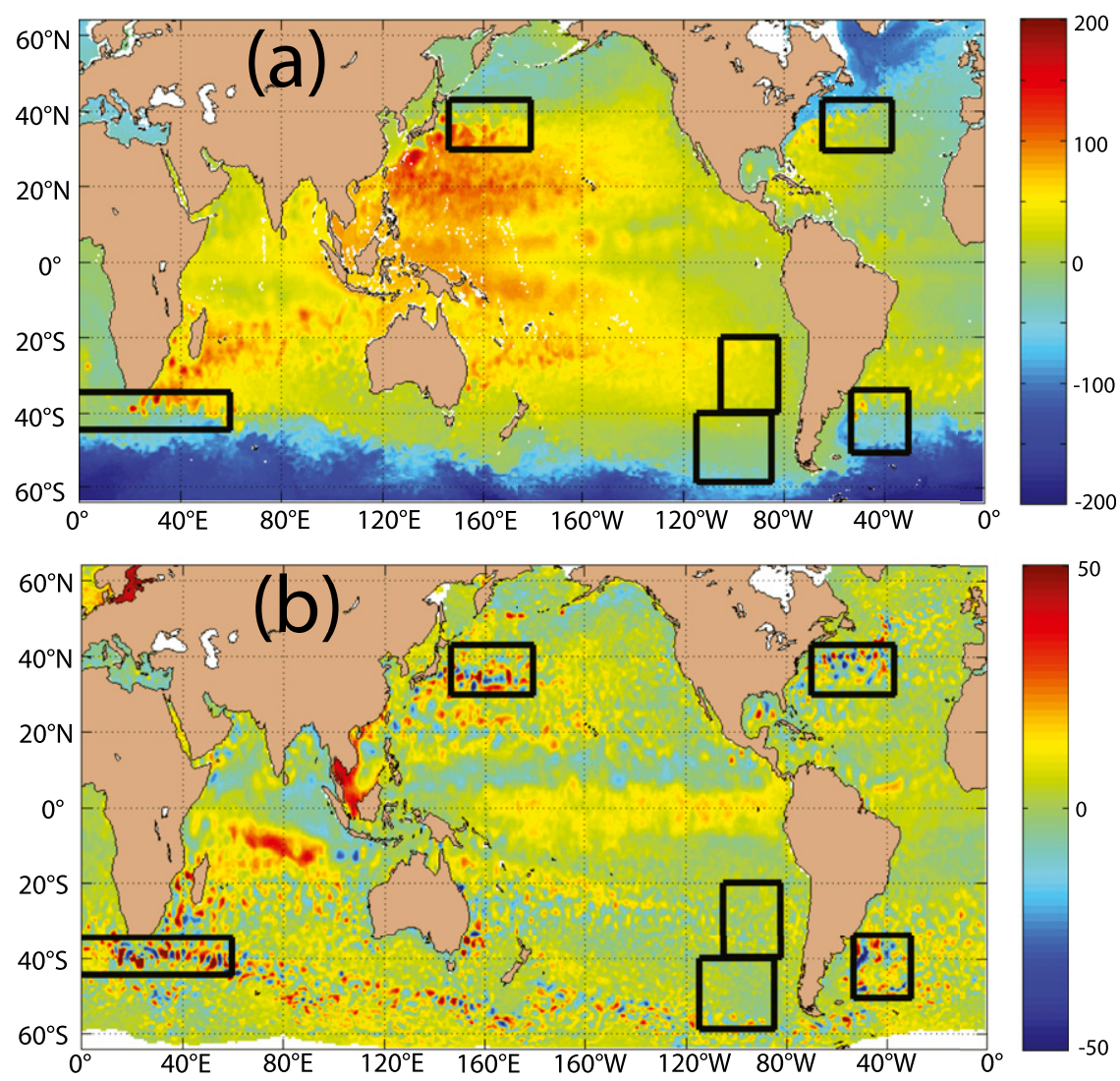

FIG. 2. Six regions used to compute spectra, spectral fluxes, and spectral transfers of surface ocean geostrophic kinetic energy from (a) $1 / 12.5^{\circ} \mathrm{HYCOM}$ output and (b) $1 / 3^{\circ}$ AVISO gridded satellite altimeter product. In (a) the regions are highlighted against the 0000 UTC 1 January 2008 snapshot of HYCOM steric sea surface height $(\mathrm{cm})$. In (b) the regions are highlighted against the AVISO 20 December 2006 snapshot of sea surface height anomaly $(\mathrm{cm})$.

so that only low-frequency (assumed geostrophic) motions remain. We analyze low-pass filtered results spaced at 1-day intervals.

Six regions of interest, over which we will compute spectral diagnostics, are shown in Fig. 2a, with a snapshot of HYCOM steric SSH serving as background. Four of the regions experience intense boundary current and mesoscale eddy activity - the Kuroshio in the western North Pacific, the Gulf Stream in the western North Atlantic, the Agulhas off the southern tip of Africa, and the Malvinas in the western South Atlantic. Two additional, relatively quiescent regions are in the southeastern Pacific-a midlatitude region and a high-latitude region that touches upon the Antarctic Circumpolar Current in its southernmost portions. Because of the removal of HYCOM land points associated with Bermuda, our Gulf Stream region is slightly narrower in east-west extent in HYCOM than in AVISO.

\section{c. Gridded satellite altimeter product}

We use 840 weekly snapshots of the two-satellite AVISO $1 / 3^{\circ}$ Mercator grid reference product (Le Traon et al. 1998; Ducet et al. 2000), beginning 14 October 1992 and ending 12 November 2008. Figure 2b displays the six regions over which we compute AVISO spectral diagnostics, shown against an example AVISO SSH anomaly snapshot. The regions correspond very closely to those utilized in the HYCOM analysis, with the greatest deviation being in the Gulf Stream as discussed above.

\section{d. Normalization of wavenumbers and frequencies}

We end this section with a brief discussion of the $x$ axes used in spectral plots throughout the paper. In the QG plots, wavenumbers are nondimensionalized by $1 / L_{d}$ and frequencies are nondimensionalized by $U / L_{d}$, where $U=\bar{u}_{1}-\bar{u}_{2}$ is the imposed mean shear. To connect the HYCOM and AVISO results with the QG results, we will display, on the HYCOM and AVISO plots, estimated oceanic values of the $1 / L_{d}$ wavenumber and $U / L_{d}$ frequency. For each of the six regions shown in Fig. 2, we choose the $L_{d}$ value at the Chelton et al. (1998) atlas grid point lying closest to the center of the region. The value of $U$ is computed as the area-weighted 
TABLE 1. Values of $L_{d}, U$, and $L_{d} / U$ (see text) for the six regions shown in Fig. 2.

\begin{tabular}{lccc}
\hline \multicolumn{1}{c}{ Region } & $L_{d}(\mathrm{~km})$ & $U\left(\mathrm{~cm} \mathrm{~s}^{-1}\right)$ & $\begin{array}{c}L_{d} / U \\
(\text { days })\end{array}$ \\
\hline $\begin{array}{l}\text { Midlatitude southeast Pacific } \\
\text { High-latitude southeast }\end{array}$ & 36 & 2.4 & 17 \\
$\quad$ Pacific & 21 & 4.6 & 5.2 \\
Agulhas & & & \\
Malvinas & 30 & 13 & 2.5 \\
Gulf Stream & 25 & 9.4 & 3.0 \\
Kuroshio & 34 & 9.6 & 4.1 \\
& 32 & 8.8 & 4.2 \\
\hline
\end{tabular}

root-mean-square of the time-mean surface velocity over the region:

$$
U=\sqrt{\frac{\iint\left(u_{\text {mean }}^{2}+v_{\text {mean }}^{2}\right) d A}{\iint d A}}
$$

where $d A$ represents an element of area, and $u_{\text {mean }}$ and $v_{\text {mean }}$ are respectively the zonal and meridional surface velocities computed from the mean dynamic topographies of Niiler et al. (2003) and Maximenko et al. (2009). We assume that the surface velocity is a reasonable proxy for the shear, that is, that velocities in the subsurface ocean are substantially weaker than the surface flow. The values of $L_{d}, U$, and $L_{d} / U$ for the six regions are given in Table 1.

\section{Theory of frequency-wavenumber spectral fluxes and transfers}

The theory of spectral transfers $T(K, \omega)$ and spectral fluxes $\Pi(K, \omega)$ in the two-layer QG model, where $K=\sqrt{k^{2}+l^{2}}$ is isotropic wavenumber and $\omega$ denotes frequency, is summarized here. We let $\hat{A}$ denote the discrete Fourier transform of an arbitrary field $A$. For example,

$$
\psi_{1}(x, y, t)=\sum_{k} \sum_{l} \sum_{\omega} \widehat{\psi_{1}}(k, l, \omega) e^{i(k x+l y+\omega t)} .
$$

Thus, the frequency-wavenumber spectrum of upperlayer kinetic energy density $\left|\nabla \psi_{1}\right|^{2} / 2$ is

$$
\mathrm{EKE}_{1}(k, l, \omega)=\frac{1}{2} K^{2} \widehat{\psi_{1}^{*}}(k, l, \omega) \widehat{\psi_{1}}(k, l, \omega),
$$

where the superscript * represents a complex conjugate.

As discussed in many sources (e.g., Salmon 1978, 1980; Hua and Haidvogel 1986; Larichev and Held 1995; LaCasce 1996), spectral transfers identify energy sources and sinks in Fourier space, which could mean wavenumber, frequency, or frequency-wavenumber space here. Positive (negative) transfer values indicate an energy source (sink). To develop a depth-averaged spectral transfer budget, we multiply the Fourier transform of the upperlayer equation [Eq. (1)] by $-\delta \widehat{\psi_{1}^{*}}(k, l, \omega) /(1+\delta)$, multiply the Fourier transform of the lower-layer equation [Eq. (2)] by $-\widehat{\psi_{2}^{*}}(k, l, \omega) /(1+\delta)$, and add the results, yielding

$$
\begin{gathered}
T_{\mathrm{KE}, 1}(k, l, \omega)+T_{\mathrm{KE}, 2}(k, l, \omega)+T_{\mathrm{APE}}(k, l, \omega)+ \\
T_{\text {forcing }}(k, l, \omega)+T_{\text {friction }}(k, l, \omega)=0 .
\end{gathered}
$$

Here the spectral transfers of upper- and lower-layer kinetic energy are

$$
T_{\mathrm{KE}, 1}(k, l, \omega)=\operatorname{Re}\left[\frac{\delta}{1+\delta} \widehat{\psi_{1}^{*}}(k, l, \omega) \widehat{J\left(\psi_{1}, \nabla^{2} \psi_{1}\right)}(k, l, \omega)\right],
$$
and

$$
T_{\mathrm{KE}, 2}(k, l, \omega)=\operatorname{Re}\left[\frac{1}{1+\delta} \widehat{\psi_{2}^{*}}(k, l, \omega) \widehat{J\left(\psi_{2}, \nabla^{2} \psi_{2}\right)}(k, l, \omega)\right],
$$

respectively, where Re denotes the real part of a complex number. ${ }^{1}$ The spectral transfers of available potential energy (APE), mean flow forcing, and bottom Ekman friction are

$$
\begin{aligned}
T_{\mathrm{APE}}(k, l, \omega)= & \operatorname{Re}\left[\frac{\delta}{(1+\delta)^{2} L_{d}^{2}} \widehat{\left(\psi_{1}-\psi_{2}\right)^{*}}(k, l, \omega)\right. \\
& \left.\times \widehat{J\left(\psi_{1}, \psi_{2}\right)}(k, l, \omega)\right], \\
T_{\text {forcing }}(k, l, \omega)= & \operatorname{Re}\left[\frac{\delta}{1+\delta} \widehat{\psi_{1}^{*}}(k, l, \omega) \widehat{\Gamma_{1}}(k, l, \omega)\right. \\
& \left.+\frac{1}{1+\delta} \widehat{\psi_{2}^{*}}(k, l, \omega) \widehat{\Gamma_{2}}(k, l, \omega)\right], \text { and }
\end{aligned}
$$

$$
T_{\text {friction }}(k, l, \omega)=-\frac{1}{1+\delta} R_{2} K^{2} \widehat{\psi_{2}^{*}}(k, l, \omega) \widehat{\psi_{2}}(k, l, \omega),
$$

\footnotetext{
${ }^{1}$ For simplicity, because stratification is not horizontally uniform in HYCOM and AVISO output, the factor $\delta /(1+\delta)$ is omitted from the HYCOM and AVISO spectral kinetic energy flux and transfer computations in this paper.
} 
respectively. The transfer terms associated with ssd, not listed in Eq. (12), are inferred as a small residual. Note that because Eq. (12) is a depth-averaged budget there are no interlayer transfers.

Spectral fluxes are defined as integrals of the spectral transfers, namely,

$$
\Pi_{\text {term }}(K, \Omega)=\int_{k^{2}+l^{2} \geq K^{2}} \iint_{\omega \geq \Omega} T_{\text {term }}(k, l, \omega) d \omega d k d l,
$$

where the subscript "term" denotes KE, 1; KE, 2; APE; forcing; or friction as appropriate. ${ }^{2}$ Integration over all frequencies $\omega$ yields spectral fluxes in isotropic wavenumber $K$ space

$$
\Pi_{\text {term }}(K)=\int_{k^{2}+l^{2} \geq K^{2}} \iint T_{\text {term }}(k, l, \omega) d \omega d k d l,
$$

while integration over all wavenumbers yields spectral fluxes in frequency space

$$
\Pi_{\text {term }}(\Omega)=\iiint_{\omega \geq \Omega} T_{\text {term }}(k, l, \omega) d \omega d k d l .
$$

\section{Analysis technique}

Spectral analysis of fields that are not periodic requires care (Priestley 1981). None of the three output fields analyzed here-from the idealized QG model, realistic model, or altimeter product-are periodic in time, nor are fields excised from the realistic model or altimeter product periodic in space. For the AVISO and 3-day low-pass filtered HYCOM output, we first convert the SSH fields $\eta$ to surface geostrophic streamfunctions, denoted by $\psi_{1}$ in analogy to the QG model, via $\psi_{1}=g \eta / f$, where $g=9.8 \mathrm{~m} \mathrm{~s}^{-2}$ is the gravitational acceleration, and $f$ is the Coriolis parameter. We remove the temporal trend and mean of $\psi_{1}$ at each spatial grid point. Following this, spatial trends and means are removed from every snapshot of $\psi_{1}$ with a two-dimensional least squares fit. To enforce periodicity in space, each $\psi_{1}$ snapshot is multiplied by a two-dimensional Tukey window having a ratio of taper to constant sections equal to 0.2 in each horizontal direction. To enforce periodicity in time, the $\psi_{1}$ time series at each spatial grid point

\footnotetext{
${ }^{2}$ In retrospect, we realize that the factor of $\delta /(1+\delta)$ was sometimes inadvertently omitted from ASFMRS plots in which the QG $\Pi_{\mathrm{KE}, 1}(\omega)$ curve was shown as a stand-alone result-that is, not as part of a depth-averaged budget. Because this omission was not made in any of the energy budget plots, it does not affect any of the conclusions of ASFMRS.
}

is multiplied by a Tukey window, also having a value of 0.2 for the ratio of taper to constant sections. ${ }^{3}$ Because the QG model is doubly periodic, and because $\psi_{1}$ has a zero time mean at each grid point, we do not employ any spatial detrending and mean removal, spatial windowing, or temporal detrending and mean removal in our QG analysis. However, the QG analysis does employ a temporal Tukey window to enforce periodicity in time. Terms involving spatial derivatives are computed using forward and inverse discrete Fourier transforms in wavenumber space. For example, $\nabla^{2} \psi_{1}$ is the inverse discrete Fourier transform of $-\left(k^{2}+l^{2}\right) \widehat{\psi_{1}}(k, l)$. For simplicity, in the HYCOM and AVISO analyses we neglect the small convergence of meridians within our regions. We use the native HYCOM and AVISO grids, rather than interpolating to regularly spaced $x-y$ grids, neglecting the order $10 \%$ variations in latitudinal spacing on Mercator grids.

Multiplication by a Tukey window, after the removal of a trend and mean, leaves behind fields having nonzero means. Therefore, some nonzero spectral energy is found in the zero frequency (wavenumber). As is commonly done, we will not include the zero frequency and wavenumber results in our plots of spectra. However, as will be seen shortly, the zero-frequency results are crucial for spectral fluxes and transfers. To display the zerofrequency (wavenumber) flux and transfer results on the semilog plots we favor throughout this paper, we move the zero-frequency (wavenumber) results to one-half of the lowest resolved nonzero frequency (wavenumber).

As described below, consideration of a joint frequencywavenumber spectral flux provides insights into the lowfrequency behavior of $\Pi_{\mathrm{KE}, 1}(\omega)$. For geostrophic flows with periodic boundary conditions, $\Pi_{\mathrm{KE}, 1}(K)$ is zero at the lowest wavenumber. Because $\Pi_{\mathrm{KE}, 1}(K, \omega)$ is a double integral over wavenumber and frequency of $T_{\mathrm{KE}, 1}(K, \omega)$, and because the order of integration should not matter, $\Pi_{\mathrm{KE}, 1}(\omega)$ must be zero at the lowest frequency. For all of the $\Pi_{\mathrm{KE}, 1}(K, \omega)$ curves in this paper, the absolute value at the lowest frequency-lowest wavenumber pair is $2 \%$ or less, often much less, of the largest absolute value occurring throughout $(K, \omega)$ space. Because $\Pi_{\mathrm{KE}, 1}(\omega)$ must be zero at the lowest frequency, the record duration impacts estimated lowfrequency $\Pi_{\mathrm{KE}, 1}(\omega)$ values. In Fig. 3a, we display the

\footnotetext{
${ }^{3}$ Note that most of the analysis in ASFMRS and Arbic et al. (2013) utilized nine overlapping Hanning windows in space (and time, in the case of ASFMRS), instead of the Tukey windows used here. As discussed in Arbic et al. (2013) overlapping Hanning windows and Tukey windows yield quantitatively similar flux estimates in high-resolution models and qualitatively similar AVISO fluxes.
} 


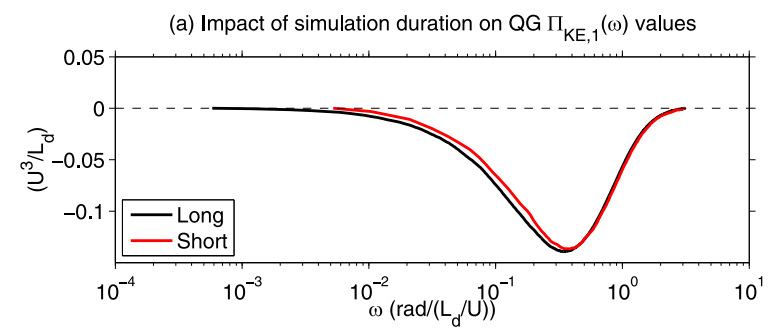

(b) Impact of detrending and highpassing on HYCOM Agulhas $\Pi_{\mathrm{KE}, 1}(\omega)$ values

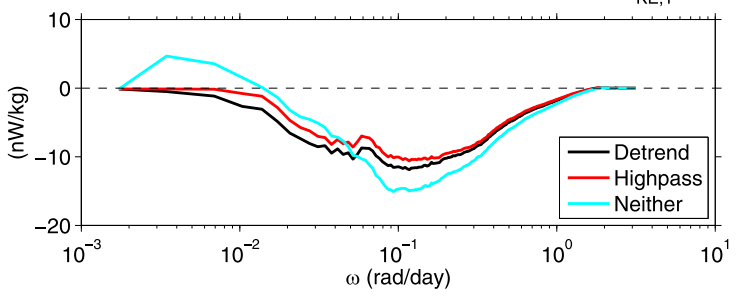

(c) Impact of detrending and highpassing on HYCOM Agulhas $T_{K E, 1}(\omega)$ values

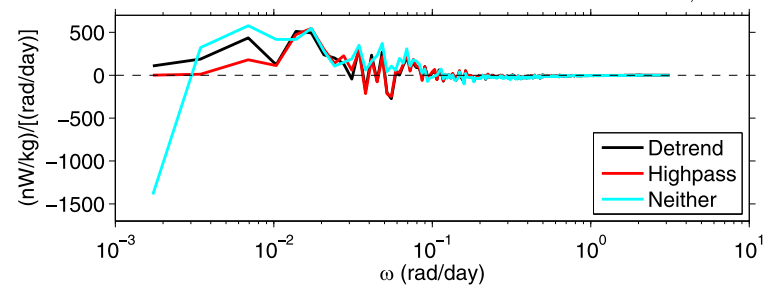

FIG. 3. (a) Spectral kinetic energy fluxes $\Pi_{\mathrm{KE}, 1}(\omega)$ in nominal QG simulation (Long; black) and shorter subset of the QG simulation (Short; red). In the axis labels of (a) and of other figures made from the QG simulation, "U" denotes the imposed mean shear $\bar{u}_{1}-\bar{u}_{2}$. (b),(c) Spectral kinetic energy fluxes and transfers $\Pi_{\mathrm{KE}, 1}(\omega)$ and $T_{\mathrm{KE}, 1}(\omega)$, respectively, in the Agulhas region of HYCOM for three analysis procedures given in legend (see text).

$\Pi_{\mathrm{KE}, 1}(\omega)$ curves from our nominal (5400 snapshot) QG simulation and from a 600-snapshot subset of this simulation (having the same temporal sampling interval). Because $\Pi_{\mathrm{KE}, 1}(\omega)$ goes to near-zero values at the low-frequency ends of both curves, and because the low-frequency end points differ between the two curves, the $\Pi_{\mathrm{KE}, 1}(\omega)$ curves separate slightly at low frequencies.

The importance of temporal detrending is illustrated in Figs. $3 \mathrm{~b}$ and $3 \mathrm{c}$. The HYCOM Agulhas region $\Pi_{\mathrm{KE}, 1}(\omega)$ curve resulting from our preferred analysis procedure (Detrend) - in which a temporal mean and linear trend is removed from each spatial grid point-is contrasted with results from two other procedures in Fig. 3b. The High-pass curve uses a high-pass to remove frequencies lower than twice the lowest resolved frequency. In the Neither curve, neither a high-pass or detrend/mean removal is employed on the time series at each model grid point. While the Neither curve displays some positive $\Pi_{\mathrm{KE}, 1}(\omega)$ values at low frequencies, the High-pass and Detrend curves display negative values (implying a cascade to lower frequencies) throughout the entire plotted frequency range. The nonlinear spectral kinetic energy fluxes $\Pi_{\mathrm{KE}, 1}(\omega)$ are strongly affected at low frequencies if temporal means and trends are not removed, consistent with the well-known effects on the spectra themselves (e.g., Priestley 1981). Because detrending is standard practice in time series analysis, we believe that the Detrend and High-pass curves in Fig. $3 \mathrm{~b}$ are more reliable than the Neither curve. Because our intuition was developed in the QG case, for which these considerations are less important as described earlier, we did not recognize the need for temporal detrending in our ASFMRS analyses, nor did we assign zero-frequency flux and transfer values to displayable nonzero frequencies. As a result the realistic model $\Pi_{\mathrm{KE}, 1}(\omega)$ curves in ASFMRS look like the Neither curve in Fig. 3b, but without the lowest frequency result-in other words, the ASFMRS realistic model $\Pi_{\mathrm{KE}, 1}(\omega)$ results show artificial positive values at low frequencies, without a drop to nearzero values at the left-hand end point. The impact of these important signal processing concerns on the HYCOM Agulhas $T_{\mathrm{KE}, 1}(\omega)$ curves is shown in Fig. 3c. At the lowest frequencies, the Neither curve has an unphysically sharp jump due to substantial energy in the (nominally) zero frequency. In the remainder of this paper, we employ the Detrend procedure.

\section{Results}

\section{a. Spectra}

We now discuss frequency-wavenumber spectra $\mathrm{EKE}_{1}$ of ocean surface kinetic energy [Eq. (11)]. The ratio of HYCOM to AVISO total kinetic energy (integrated over all frequencies and wavenumbers) is of order one -0.85 in the Kuroshio region and between 1.1 and 1.3 in the other five regions. Frequency-wavenumber spectra for the QG model and for HYCOM and AVISO results in the Agulhas region, which is reasonably representative, are shown in Fig. 4. The spectra display several visual similarities, including a tendency for highwavenumber energy to be nearly independent of frequency, a tendency for a flat frequency spectrum at low frequencies, and a tendency for lower-wavenumber energy to be associated with lower frequencies. This latter feature is seen as a diagonal "cut" in the right-hand side of each subplot (implying small low-wavenumber energy at high frequencies). The spectra are visually "wider" in frequency space than in wavenumber space. Substantial values of $\mathrm{EKE}_{1}$ are seen over a decade or less in wavenumber, but over two or more decades in frequency. Because $\mathrm{EKE}_{1}$ rolls off at the lowest wavenumbers, analyses in larger domains would not see a continuation of substantial $\mathrm{EKE}_{1}$ values out to yet lower wavenumbers. 
(a) $Q G$

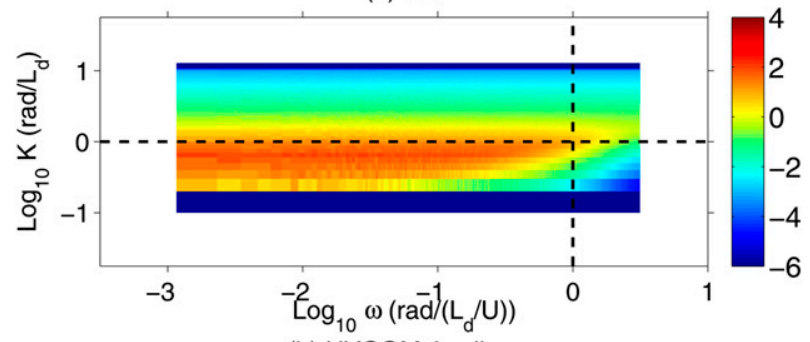

(b) HYCOM Agulhas

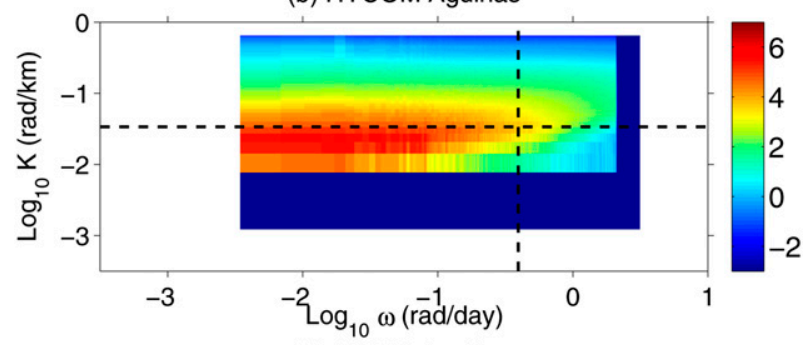

(c) AVISO Agulhas

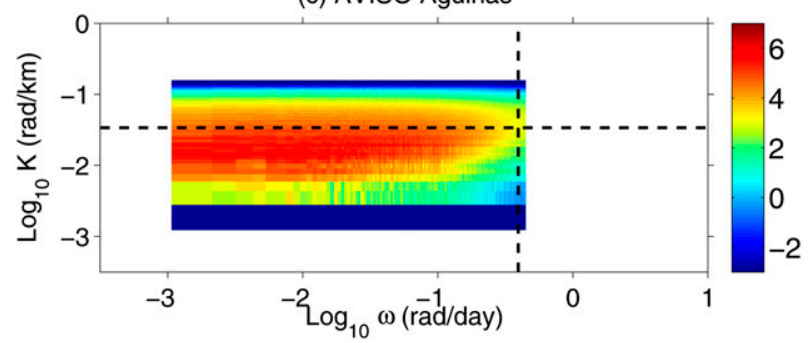

FIG. 4. Wavenumber-frequency $(K-\omega)$ spectrum $\operatorname{EKE}_{1}(K, \omega)$ of $\left|\nabla \psi_{1}\right|^{2} / 2$ (kinetic energy) from the (a) upper layer of the idealized two-layer QG simulation and from Agulhas region of ocean surface in (b) HYCOM and (c) AVISO. Units of spectra are $\log _{10}$ of $0.5\left(\bar{u}_{1}-\bar{u}_{2}\right)^{2} /\left(\left[\operatorname{rad} / L_{d}\right]\left\{\mathrm{rad} /\left[L_{d} /\left(\bar{u}_{1}-\bar{u}_{2}\right)\right]\right\}\right)$ in (a) and $\log _{10}$ of $\left(\mathrm{cm} \mathrm{s}^{-1}\right)^{2} /\left[\left(\operatorname{rad~day}^{-1}\right)\left(\mathrm{rad} \mathrm{km}^{-1}\right)\right]$ in (b) and (c). Dashed horizontal and vertical lines respectively represent the deformation wavenumber $1 \mathrm{rad} / L_{d}$ and $1 \mathrm{rad} /\left(L_{d} / U\right)$, where $U$ is the mean shear (see text). The $x$ and $y$ axes, and the colorbar, in the HYCOM subplot (b) are identical to those in the AVISO subplot (c). The $x$ and $y$ axes, and the colorbar, in the QG subplot (a) cover the same number of decades as do those in the HYCOM and AVISO subplots.

In contrast, because of the flatness of $\mathrm{EKE}_{1}$ at low frequencies, the record duration determines the range of frequencies over which substantial $\mathrm{EKE}_{1}$ values are seen. The QG record is the longest of the three shown in Fig. $4-86 \mathrm{yr}$ if one takes typical values of $L_{d}=50 \mathrm{~km}$ and $U=10 \mathrm{~cm} \mathrm{~s}^{-1}$-hence, it has the largest width in frequency. The asymmetry between spectral widths in wavenumber versus frequency will be seen throughout this paper.

To further investigate the association of low-frequency and low-wavenumber energy, we compute the energycontaining wavenumbers $K_{E}(\omega)$, and energy-containing frequencies $\omega_{E}(K)$, defined as first moments (centroids) of the spectrum, namely,

$$
K_{E}(\omega)=\frac{\iint \sqrt{k^{2}+l^{2}} \operatorname{EKE}_{1}(k, l, \omega) d k d l}{\iint \operatorname{EKE}_{1}(k, l, \omega) d k d l},
$$

(for a fixed $\omega$ ) where $\operatorname{EKE}_{1}(k, l, \omega)$ is the kinetic energy spectrum [Eq. (11)], and

$$
\omega_{E}(K)=\frac{\int \omega \operatorname{EKE}_{1}(K, \omega) d \omega}{\int \operatorname{EKE}_{1}(K, \omega) d \omega}
$$

for a fixed isotropic wavenumber $K$. A general association between low-wavenumber and low-frequency energy is found in plots of $\omega_{E}(K)$ and $K_{E}(\omega)$ for the models and altimeter maps (Fig. 5). The correspondence is not simple, and the plots are noisy, especially near the Nyquist wavenumbers and frequencies. Note that a general association of low-frequency energy with low wavenumbers does not preclude some association of low-frequency energy with high wavenumbers. As an example, Wortham (2013) points out that the 18-yr mean of AVISO SSH contains considerable smallscale (high wavenumber) structure.

The frequency-wavenumber spectra shed light on the limitations of the Taylor (1938) hypothesis with respect to geostrophic turbulence. The Taylor hypothesis states that when turbulent velocities are small compared to a mean flow $V$, the wavenumber and frequency domains are related simply, via $\omega=V k$. Variants of Taylor's hypothesis have taken $V$ to be a root-mean-square eddy speed (Tennekes 1975) or an eddy propagation speed (Ferrari and Wunsch 2010). If the Taylor hypothesis held exactly, all of the energy in the frequencywavenumber spectra would be along a line with slope determined by $V$. In this ideal case, integrating in either wavenumber or frequency would yield identical results, because the same point on the line would be picked up in either case. In fact substantial energy does lie along nearly straight lines in frequency-wavenumber spectra (e.g., Wunsch 2009, 2010; Early et al. 2011; Wortham 2013). However, for every wavenumber there is a spectrum of energy spread among all frequencies, and for every frequency there is a spectrum of energy spread among all wavenumbers (Fig. 4). The asymmetries between spectra in the two axis directions imply that the frequency and wavenumber spectra, as well as their slopes, are not easily relatable. In ASFMRS we noted further that the difference in the slopes of streamfunction variance (not shown in the present paper) and geostrophic velocity variance (kinetic energy) in the frequency domain is -0.8 , not -2 as it is in the wavenumber domain and as it therefore would be in 

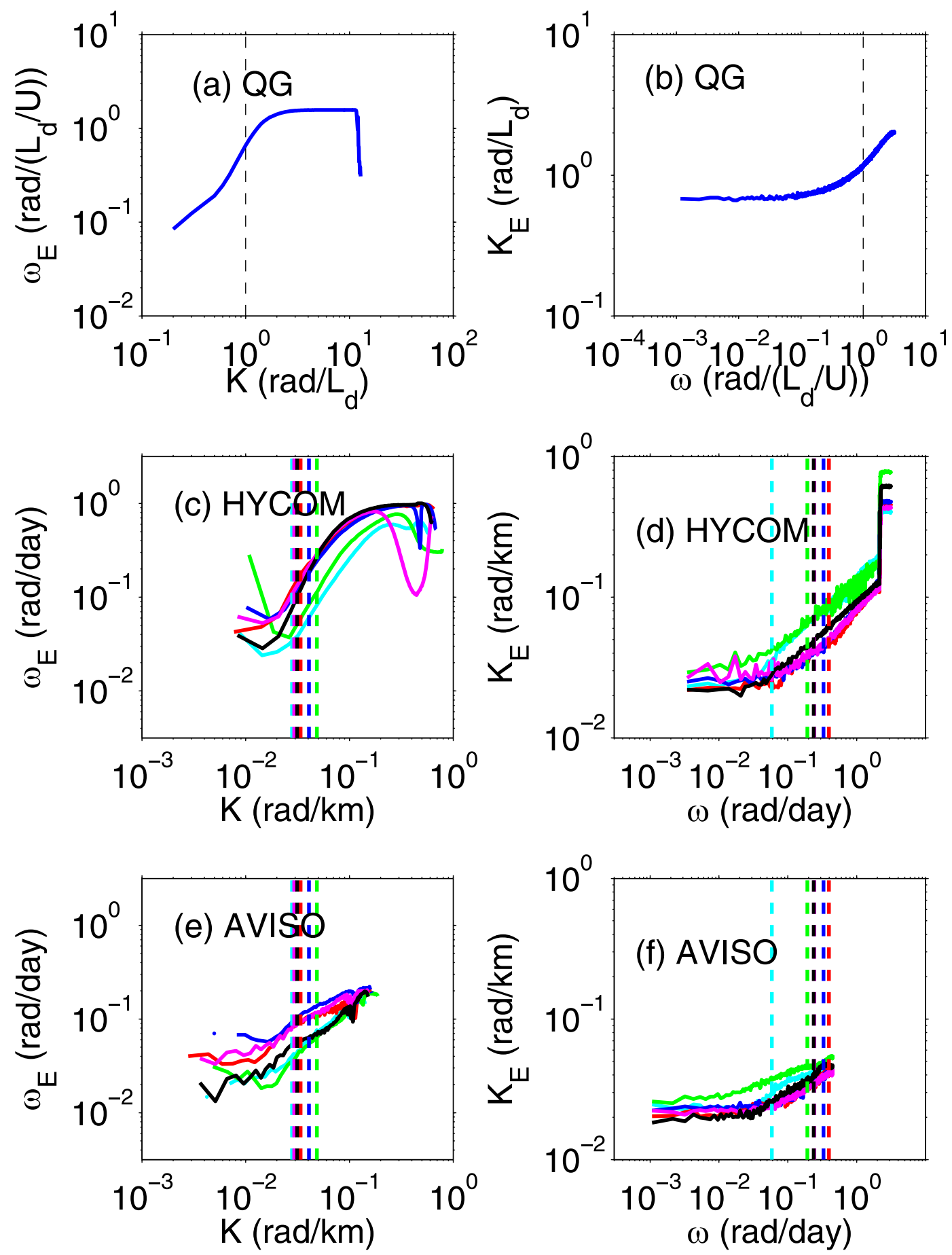

FIG. 5. (left) Centroids $\omega_{E}(K)$ of kinetic energy spectrum vs wavenumber $K$. (right) Centroids $K_{E}(\omega)$ of kinetic energy spectrum vs frequency $\omega$. Results shown for (a),(b) upper layer of idealized two-layer QG model, (c),(d) ocean surface in HYCOM, and (e),(f) ocean surface in AVISO. In (c),(d),(e), and (f), colors represent regions shown in Fig. 2. Cyan is midlatitude southeast Pacific, green is high-latitude southeast Pacific, red is Agulhas, blue is Malvinas, magenta is Gulf Stream, and black is Kuroshio. Dashed vertical lines in (a),(c), and (e) represent the deformation wavenumber $1 \mathrm{rad} / L_{d}$ (see text). Dashed lines in (b),(d), and (f) represent $1 \mathrm{rad} /\left(L_{d} / U\right)$, where $U$ is the mean shear (see text). 
the frequency domain if the Taylor hypothesis held precisely.

Wavenumber spectra, integrated over all frequencies, are shown in Fig. 6 for the QG simulation and for Agulhas HYCOM and AVISO output. Slopes of $-5 / 3$ and -3 , the predicted values for energy and enstrophy cascades, respectively (e.g., Vallis 2006), are indicated by dashed slanted green lines. The QG spectrum (Fig. 6a) drops off steeply at the highest wavenumbers due to the action of the wavenumber filter. A long band, covering wavenumbers slightly lower than the $1 / L_{d}$ wavenumber up to higher wavenumbers just before the action of the wavenumber filter, sees a slope somewhat steeper than -3 . A narrow band of wavenumbers, lying at scales somewhat larger than $L_{d}$, exhibits a shallower slope and contains the largest $\operatorname{EKE}_{1}(K)$ values. At the lowest wavenumbers the spectrum rolls off quickly to relatively low values. The HYCOM and AVISO wavenumber spectra (Fig. 6b) resemble the QG spectrum in many respects. For instance, the $L_{d}$ scale again lies near the low-wavenumber end of the steep-slope band, and the spectra roll off rapidly at the lowest wavenumbers. The HYCOM and AVISO spectra lie close together over much of the range displayed, but at higher wavenumbers the AVISO spectrum rolls off rapidly due to the filtering inherent in the construction of AVISO. At high wavenumbers, the HYCOM spectrum is affected by scale-selective dissipation acting near the model grid spacing, as well as by the 3-day low-pass filter; in general, high frequencies are associated with high wavenumbers. In this location the HYCOM wavenumber spectral slope lies reasonably close to the predicted -3 value for QG “interior mode” turbulence. Richman et al. (2012), Sasaki and Klein (2012), and references therein provide detailed discussions of wavenumber spectra in realistic models.

Frequency spectra, integrated over all wavenumbers, are displayed in Fig. 7a for the QG simulation and in Fig. $7 \mathrm{~b}$ for Agulhas HYCOM and AVISO output. A slope of -2 , the value predicted by energy cascade arguments applied to frequency space (Tennekes and Lumley 1972), is indicated by dashed slanted green lines. All three spectra are flat at lower frequencies and steeply sloped at higher frequencies, with the $L_{d} / U$ time scale lying in the middle of the steep slope band. This basic shape is found in frequency spectra computed from current meter records (e.g., Richman et al. 1977; Ferrari and Wunsch 2010; Wortham 2013) and in previous studies using altimeter products (e.g., Wunsch 2009, 2010; Wortham 2013). The HYCOM and AVISO spectra are close together over much of the range shown, with the largest deviations seen at high frequency where the filtering inherent in the construction of AVISO
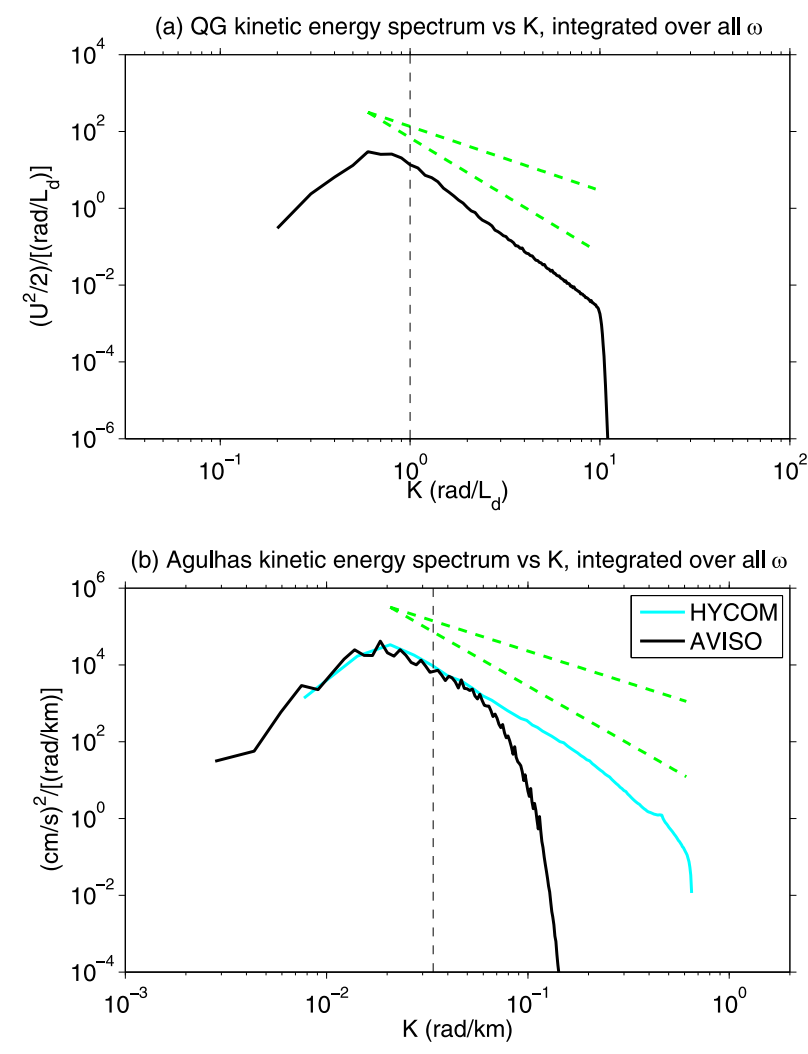

FIG. 6. Wavenumber domain kinetic energy spectra $\operatorname{EKE}_{1}(K)$, integrated over all $\omega$. (a) Upper-layer kinetic energy spectra in idealized two-layer QG simulation. Dashed vertical line denotes the deformation wavenumber $1 \mathrm{rad} / L_{d}$. (b) Ocean surface kinetic energy spectra in HYCOM and AVISO Agulhas region output. Dashed vertical line denotes the deformation wavenumber $1 \mathrm{rad} / L_{d}$ for the Agulhas region (see text). Dashed slanted green lines indicate slopes of $-5 / 3$ and -3 .

yields a steep rolloff. HYCOM frequency spectra slopes over the 14-68-day band (indicated by red vertical lines in Fig. 7b), which lies within the steep slope part of the spectrum, are between -1.6 and -2.1 over the six regions. This range is close to the -1.6 to -2.2 range seen over the same six regions in the NLOM realistic simulations analyzed by ASFMRS and to the -1.8 slope in the steeper part of the QG spectrum (ASFMRS), as well as to the -2 slopes seen in Wortham (2013)'s analyses of models, along-track satellite altimeter data, and in situ observations.

\section{b. Spectral fluxes}

We discuss spectral fluxes before spectral transfers because fluxes are smoother and hence provide an easier platform for comparison of the QG, HYCOM, and AVISO results. The inverse kinetic energy cascade toward lower wavenumbers, indicated by negative values along the middle of the wavenumber axes, is readily apparent in the QG, Agulhas HYCOM, and Agulhas 
(a) QG kinetic energy spectrum vs $\omega$, integrated over all K

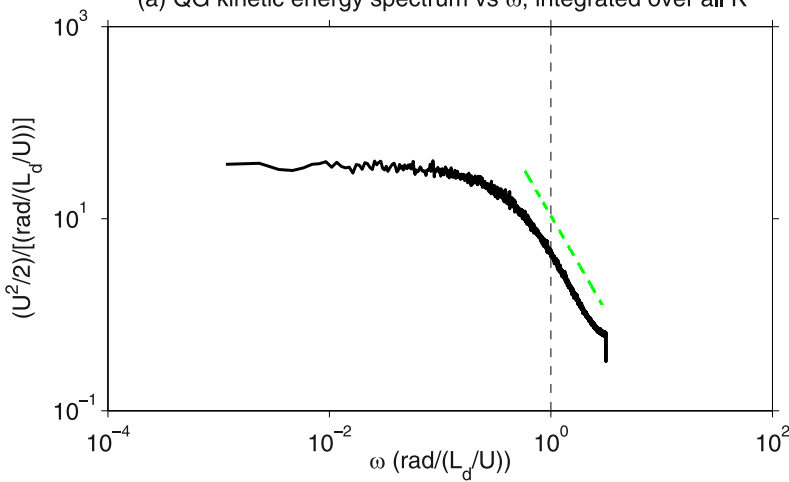

(b) Agulhas kinetic energy spectrum vs $\omega$, integrated over all $\mathrm{K}$

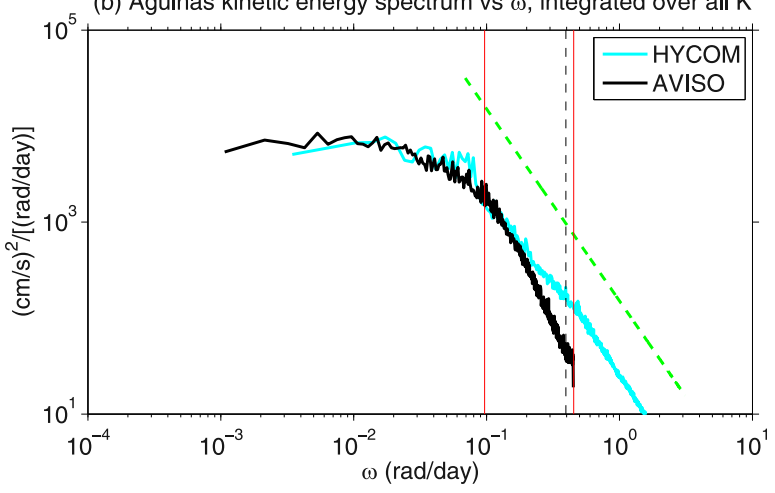

FIG. 7. Frequency domain kinetic energy spectra $\operatorname{EKE}_{1}(\omega)$ integrated over all $K$. (a) Upper-layer kinetic energy spectra in idealized two-layer QG simulation. Dashed vertical line denotes the frequency $1 \mathrm{rad} /\left(L_{d} / U\right)$, where $U$ is the imposed mean shear $\bar{u}_{1}-\bar{u}_{2}$. (b) Ocean surface kinetic energy spectra in HYCOM and AVISO Agulhas region output. Dashed vertical black line denotes the frequency $1 \mathrm{rad} /\left(L_{d} / U\right)$, where $L_{d}$ is the deformation radius, and $U$ is the roughly estimated mean shear for the Agulhas region (see text). Red vertical lines in (b) indicate periods of 14 and 68 days. Slopes of the HYCOM frequency spectra computed over this band are discussed in the text. Dashed slanted green lines indicate slope of -2 .

AVISO $\Pi_{\mathrm{KE}, 1}(K, \omega)$ maps shown in Fig. 8 . The positive AVISO flux values at higher wavenumbers are discussed extensively in Arbic et al. (2013). The negative $\Pi_{\mathrm{KE}, 1}$ values along the frequency axes of Fig. 8 imply a nonlinear kinetic energy cascade toward lower frequencies alongside the inverse cascade to lower wavenumbers. As with the spectra, the spectral fluxes are wider along frequency axes than along wavenumber axes. As will be seen shortly, with the exception of the midlatitude southeast Pacific, HYCOM $\Pi_{\mathrm{KE}, 1}$ results in other regions are similar to the HYCOM Agulhas $\Pi_{\mathrm{KE}, 1}$ results, while AVISO results are less consistent between regions than the HYCOM results are.

The depth-averaged budget of QG spectral fluxes is shown versus wavenumber in Fig. 9a and versus frequency in Fig. 9b. In both subplots the lower-layer (a) $Q G$

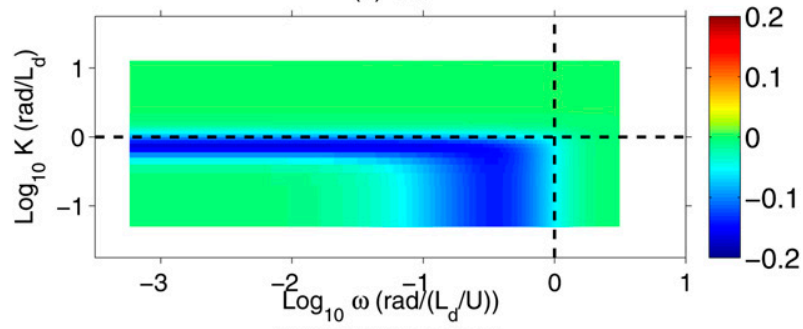

(b) HYCOM Agulhas

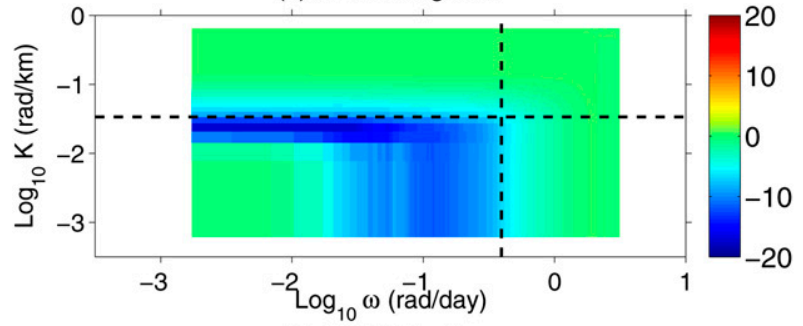

(c) AVISO Agulhas

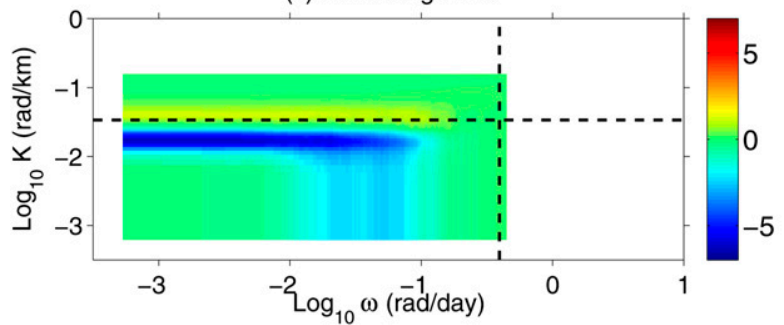

FIG. 8. Nonlinear spectral kinetic energy flux $\Pi_{\mathrm{KE}, 1}(K, \omega)$ in (a) upper layer of idealized two-layer QG simulation and from Agulhas region of (b) HYCOM and (c) AVISO ocean surface output. The units are $\left[\left(\bar{u}_{1}-\bar{u}_{2}\right)^{3} / L_{d}\right]$ in (a) and $(\mathrm{nW} / \mathrm{kg})$ in (b) and (c). Dashed horizontal and vertical lines are as in Fig. 4. The $x$ and $y$ axes are also as in Fig. 4.

kinetic energy and residual terms are much smaller than the other terms. Figure $9 \mathrm{~b}$ indicates that the kinetic energy cascade toward lower frequencies is approximately balanced by an APE cascade toward higher frequencies, similar to the wavenumber-space balance between inverse kinetic energy cascades and forward APE cascades (Salmon 1980) seen in Fig. 9a. The positive (negative) value of $\Pi_{\text {forcing }}\left(\Pi_{\text {friction }}\right)$ at the lowest wavenumber and lowest frequency implies that the forcing (friction) adds energy to (removes energy from) the system. Significant $\Pi_{\mathrm{KE}, 1}$ values cover about one decade in wavenumber and two decades in frequency.

The nonlinear surface ocean spectral kinetic energy fluxes $\Pi_{\mathrm{KE}, 1}(K)$ and $\Pi_{\mathrm{KE}, 1}(\omega)$ are shown across all six regions in Figs. 10 and 11, respectively, for unadulterated HYCOM output, HYCOM output that has undergone further filtering, and AVISO output. Spatial filtering, temporal filtering, and combined spatial and temporal filtering are performed on HYCOM output to ascertain the effects of the filtering inherent in the 
(a) QG $\Pi(K)$ budget

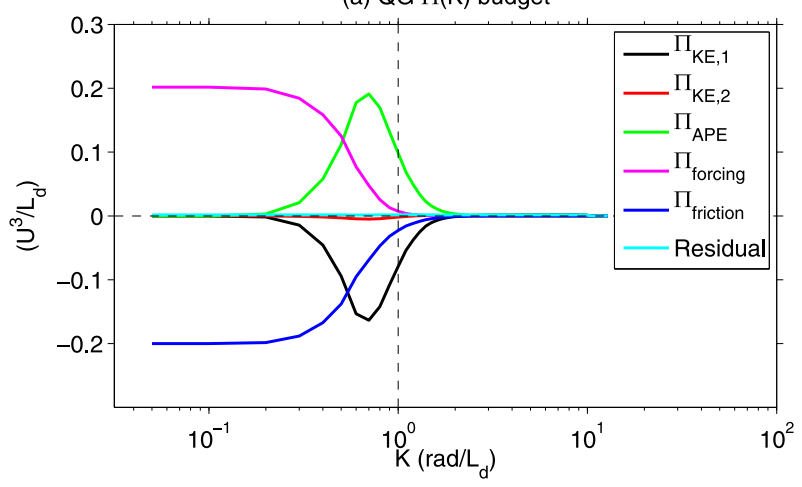

(b) QG $\Pi(\omega)$ budget

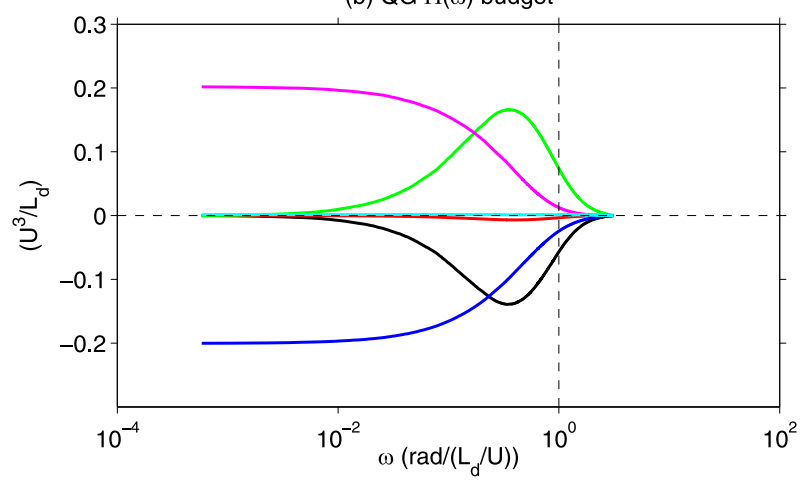

FIG. 9. (a) Depth-averaged budget of wavenumber domain spectral fluxes $\Pi(K)$ in QG model. Dashed vertical line represents the deformation wavenumber $1 \mathrm{rad} / L_{d}$. (b) Depth-averaged budget of frequency domain spectral fluxes $\Pi(\omega)$ in QG model. Dashed vertical line represents the frequency $1 \mathrm{rad} /\left(L_{d} / U\right)$, where $U$ is the imposed mean shear $\bar{u}_{1}-\bar{u}_{2}$. Budget terms identified by legend shown in (a).

creation of AVISO products on spectral flux estimates. The filtering is applied to HYCOM output after the model has been run. We utilize Blackman filters, with filter scales inspired by the spatial and temporal scales in the AVISO product. Because the AVISO product is put out every 7 days, on a $1 / 3^{\circ}$ Mercator grid, we choose a temporal Blackman filter that goes to zero 7 days from the central time and a spatial Blackman filter that goes to zero $37 \mathrm{~km}\left(1 / 3^{\circ}\right.$ latitude) from the central grid point.

As in previous studies (Scott and Wang 2005; Scott and Arbic 2007; Schlösser and Eden 2007; Tulloch et al. 2011; ASFMRS; Arbic et al. 2013), negative $\Pi_{\mathrm{KE}, 1}(K)$ values, representing inverse kinetic energy cascades to lower wavenumbers, are ubiquitous (seen in every subplot of Fig. 10). As noted in Scott and Wang (2005), the deepest negative value of the spectral fluxes $\Pi(K)$ varies by two orders of magnitude across ocean basins. As in Arbic et al. (2013), we find that filtering model output diminishes the deepest negative $\Pi_{\mathrm{KE}, 1}(K)$ values and shifts the model $\Pi_{\mathrm{KE}, 1}(K)$ curves toward lower wavenumbers.
The filtered HYCOM $\Pi_{\mathrm{KE}, 1}(K)$ curves lie closer to the AVISO curves than the unadulterated HYCOM curves do, especially when both spatial and temporal filtering are employed. Exact matches between filtered HYCOM and AVISO curves cannot be expected, not only because of model limitations but also because our filtering procedure mimics the AVISO construction procedure only qualitatively.

The negative $\Pi_{\mathrm{KE}, 1}(\omega)$ values seen across the entire frequency range in the unadulterated HYCOM results of Figs. 11b-f are consistent with QG behaviors and demonstrate that in those regions nonlinearities drive a kinetic energy cascade toward lower frequencies. As noted in section 4 , the removal of temporal trends and means is crucial for obtaining this consistency with QG results across the entire plotted frequency range. As in the $Q G$ results, significant $\Pi_{\mathrm{KE}, 1}$ values take place over about two decades in frequency, but only about one decade in wavenumber. In the midlatitude southeast Pacific (Fig. 11a), $\Pi_{\mathrm{KE}, 1}(\omega)$ in unadulterated HYCOM is negative for higher frequencies but positive for lower frequencies, in contrast to HYCOM behaviors in other regions and to QG behaviors. An explanation for the positive $\Pi_{\mathrm{KE}, 1}(\omega)$ values at low frequencies in the HYCOM midlatitude southeast Pacific remains to be found. We do note that in the same region the AVISO $\Pi_{\mathrm{KE}, 1}(\omega)$ values are also positive over a similar range of (lower) frequencies. In like manner to the wavenumber domain behaviors, filtering diminishes the negative $\Pi_{\mathrm{KE}, 1}(\omega)$ lobes in the HYCOM results and shifts $\Pi_{\mathrm{KE}, 1}(\omega)$ curves toward lower frequencies. The AVISO spectral fluxes $\Pi_{\mathrm{KE}, 1}(\omega)$ also tend to be diminished and shifted toward lower frequencies relative to HYCOM. In general, the filtered HYCOM fluxes lie closer to the AVISO fluxes than do the unadulterated HYCOM fluxes. In contrast to the AVISO wavenumber fluxes $\Pi_{\mathrm{KE}, 1}(K)$, clearly identifiable negative lobes are not seen in the AVISO $\Pi_{\mathrm{KE}, 1}(\omega)$ curves across all six regions. The discrepancies between the signs of AVISO and unadulterated HYCOM frequency domain spectral fluxes could be due to important physics missing from HYCOM or to the filtered nature of the AVISO product. Filtering shifts the HYCOM $\Pi_{\mathrm{KE}, 1}(\omega)$ values from negative to positive over some frequencies and in some regions (see Figs. 11d, 11e, and especially Fig. 11f). Finally, we note that the position of the $L_{d}$ and $L_{d} / U$ scales, respectively, relative to the shapes of the $\Pi_{\mathrm{KE}, 1}$ curves, are similar in the unadulterated HYCOM results (black curves in Figs. 10 and 11) and QG results (black curves in Fig. 9).

\section{c. Spectral transfers}

The nonlinear spectral kinetic energy transfers $T_{\mathrm{KE}, 1}(K, \omega)$ from the upper layer of the QG simulation, 
HYCOM

Filtered in space

Filtered in time

Filtered in space and time

AVISO

(a) Midlat SE Pacific

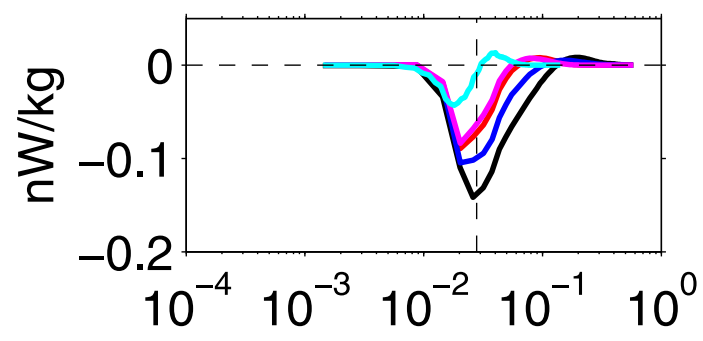

(c) Agulhas

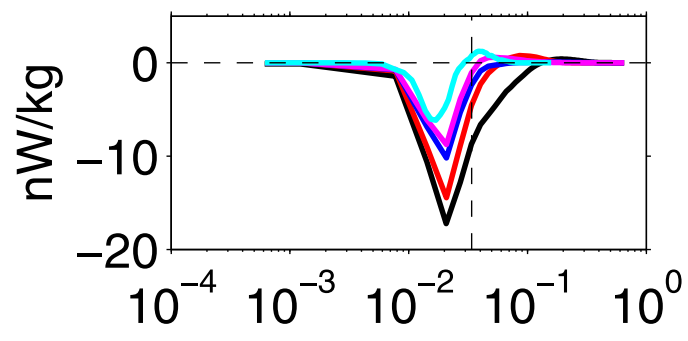

(e) Gulf Stream

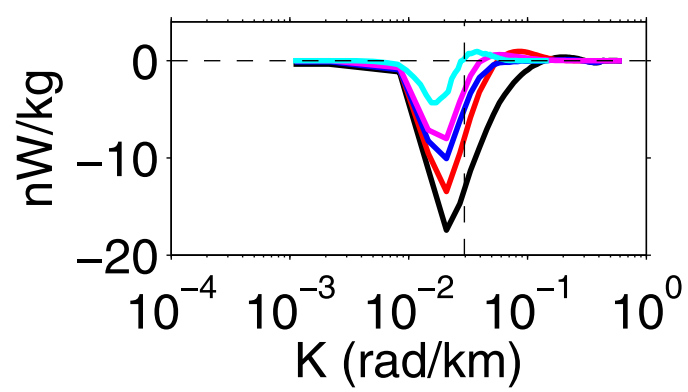

(b) Highlat SE Pacific

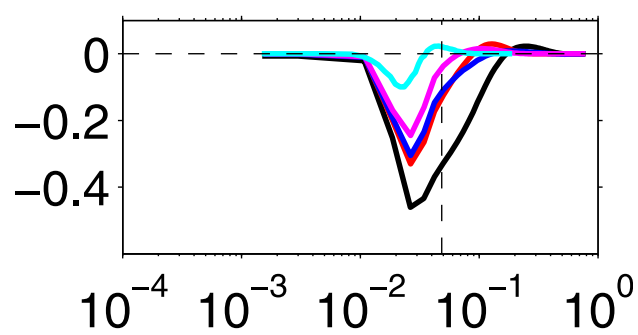

(d) Malvinas

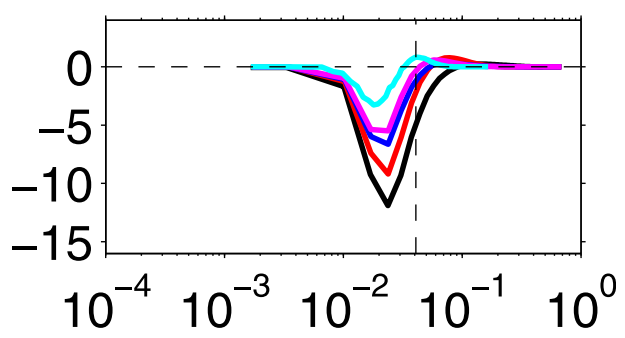

(f) Kuroshio

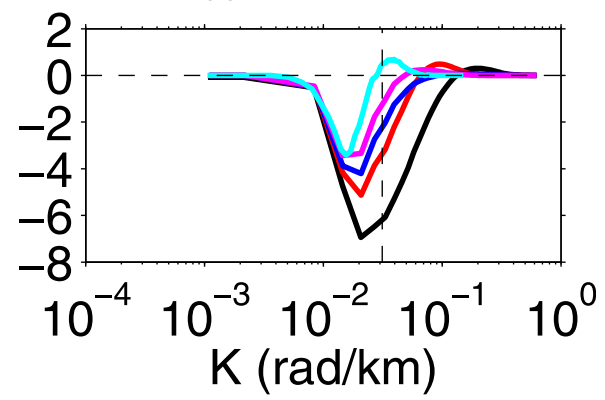

FIG. 10. Nonlinear surface ocean spectral kinetic energy flux $\Pi_{\mathrm{KE}, 1}(K)$ in wavenumber $K$ space, for six regions shown in Fig. 2, computed from unadulterated HYCOM output, spatially filtered HYCOM output, temporally filtered HYCOM output, HYCOM output filtered in both space and time, and AVISO. See text for details of spatial and temporal filters. Dashed vertical lines denote the region-dependent deformation wavenumbers $1 \mathrm{rad} / L_{d}(\mathrm{see}$ text).

and from Agulhas HYCOM and AVISO ocean surface output, are shown in Fig. 12. To display the smaller negative transfers clearly while using a symmetric colorbar, we choose a colorbar range that saturates for the larger positive transfers. The transfers are noisier than the fluxes because the fluxes are integrals of the transfers. However, as with the spectra and spectral fluxes, it is still clear that the spectral transfers are wider in frequency 


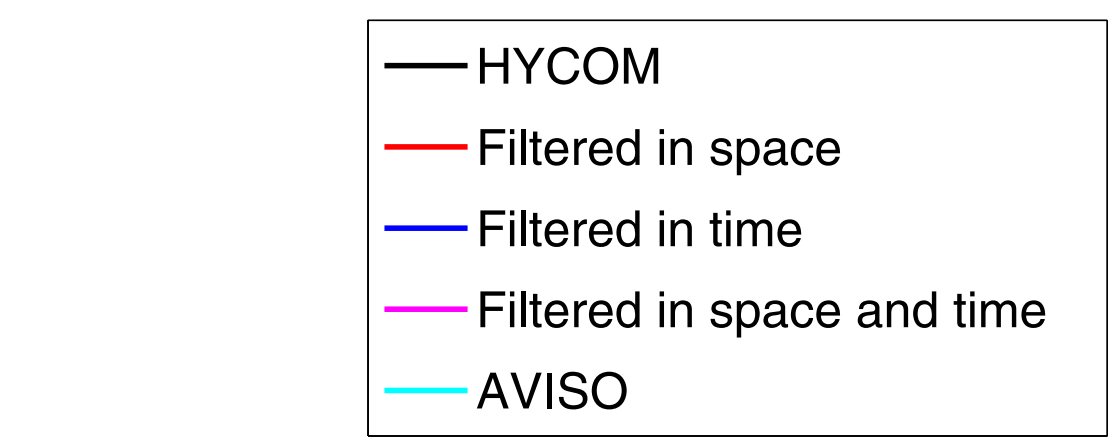

(a) Midlat SE Pacific

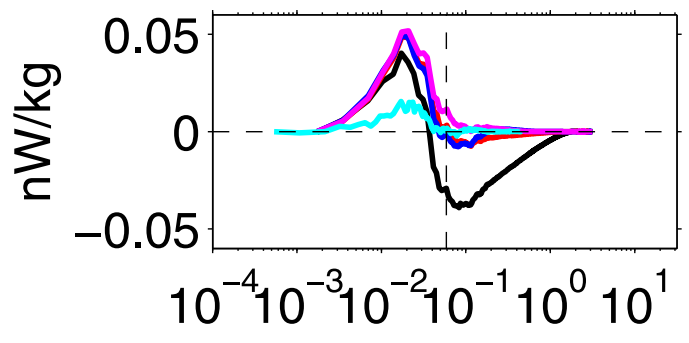

(c) Agulhas

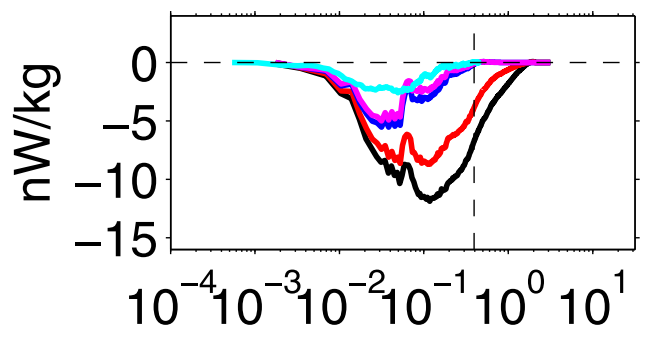

(e) Gulf Stream

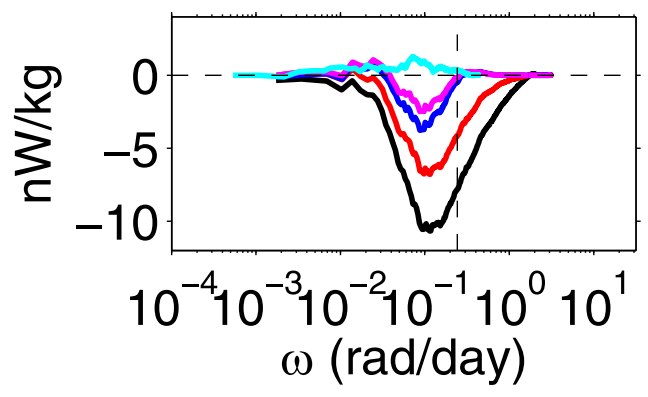

(b) Highlat SE Pacific

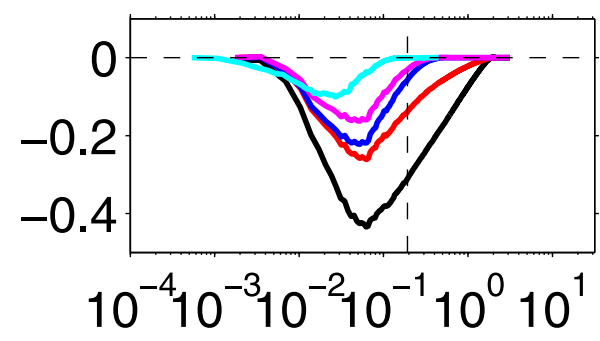

(d) Malvinas

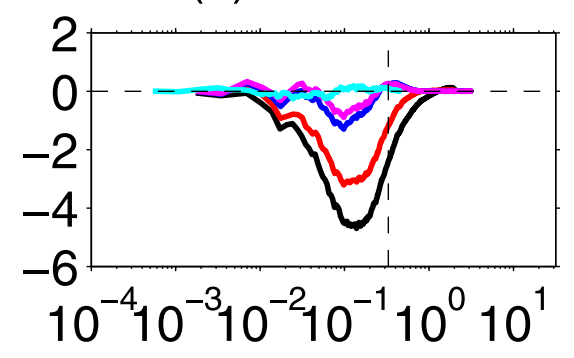

(f) Kuroshio

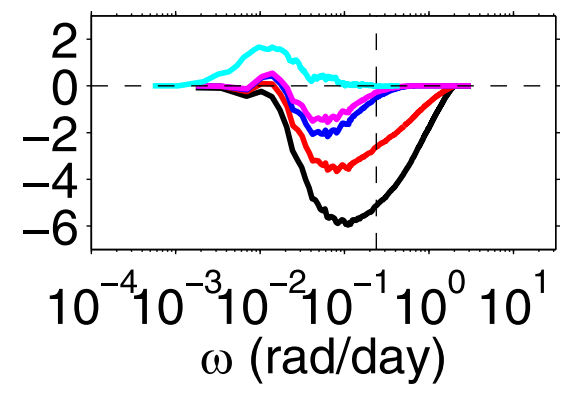

FIG. 11. As in Fig. 10, but for nonlinear surface ocean spectral kinetic energy fluxes $\Pi_{\mathrm{KE}, 1}(\omega)$ in frequency $(\omega)$ space. Dashed vertical lines denote the region-dependent frequencies $1 \mathrm{rad} /\left(L_{d} / U\right)$, where $L_{d}$ is the deformation radius and $U$ is the mean shear (see text).

space than in wavenumber space. Substantial $T_{\mathrm{KE}, 1}$ values cover about one decade in wavenumber, meaning that a narrow range of wavenumbers is engaged in nonlinear transfer. The range is wider-two or more decades depending on record duration-for frequency.
The QG transfers (Fig. 12a) are generally negative for higher wavenumbers and positive for lower wavenumbers. At higher frequencies the QG transfers are negative, while at lower frequencies the sign of the transfer depends on wavenumber. The diagonal cut in 
(a) QG

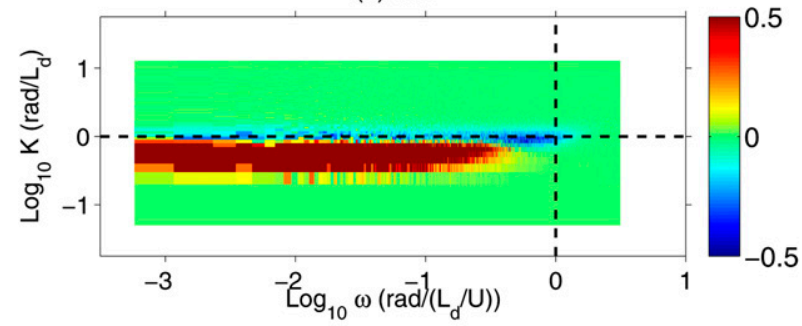

(b) HYCOM Agulhas

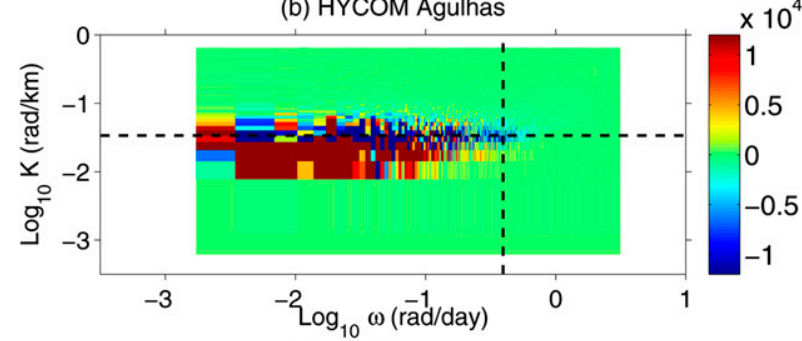

(c) AVISO Agulhas

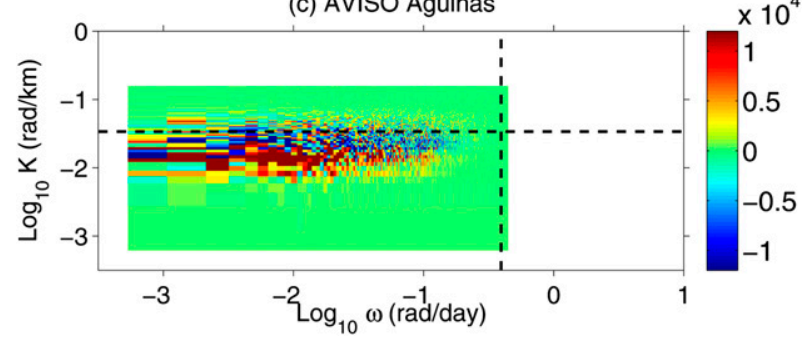

FIG. 12. Nonlinear spectral kinetic energy transfer $T_{\mathrm{KE}, 1}(K, \omega)$ in (a) upper layer of idealized two-layer QG simulation and from Agulhas region of (b) HYCOM and (c) AVISO ocean surface output. The units are $\left[\left(\bar{u}_{1}-\bar{u}_{2}\right)^{3} / L_{d}\right] /\left(\left[\mathrm{rad} / L_{d}\right]\left\{\mathrm{rad} /\left[L_{d} /\right.\right.\right.$ $\left.\left.\left.\left(\bar{u}_{1}-\bar{u}_{2}\right)\right]\right\}\right)$ in (a) and $\left(\mathrm{nW} \mathrm{kg}^{-1}\right) /\left[\left(\operatorname{rad~day}^{-1}\right)\left(\mathrm{rad} \mathrm{km}^{-1}\right)\right]$ in (b) and (c). Dashed horizontal and vertical lines are as in Fig. 4. The $x$ and $y$ axes are also as in Fig. 4.

the right-hand side of the QG spectrum (Fig. 4a) is visible in the QG transfers as well. The HYCOM and AVISO transfers (Figs. 12b and 12c, respectively) are somewhat similar to the QG transfers, but are much noisier.

The QG wavenumber domain, upper-layer kinetic energy transfer $T_{\mathrm{KE}, 1}(K)$ is shown as a black curve in Fig. 13a. Consistent with earlier work (e.g., Salmon 1978, 1980; Hua and Haidvogel 1986; Larichev and Held 1995; LaCasce 1996), a negative lobe is seen in $T_{\mathrm{KE}, 1}(K)$ near the $1 / L_{d}$ wavenumber (vertical dashed line), implying that nonlinearities act as a sink of kinetic energy near the $L_{d}$ scales associated with baroclinic instability. The positive lobe at lower wavenumbers implies that nonlinearities deposit the energy removed at near- $L_{d}$ scales into lower wavenumbers. Figure 13a also displays the other terms in the spectral transfer budget [Eq. (12)]. The nonlinear upper-layer kinetic energy and APE transfers are the largest terms in the $T(K)$ budget and roughly balance each other out as discussed in Salmon (1980). The forcing and friction terms are important (a) QG T(K) budget, integrated over all $\omega$

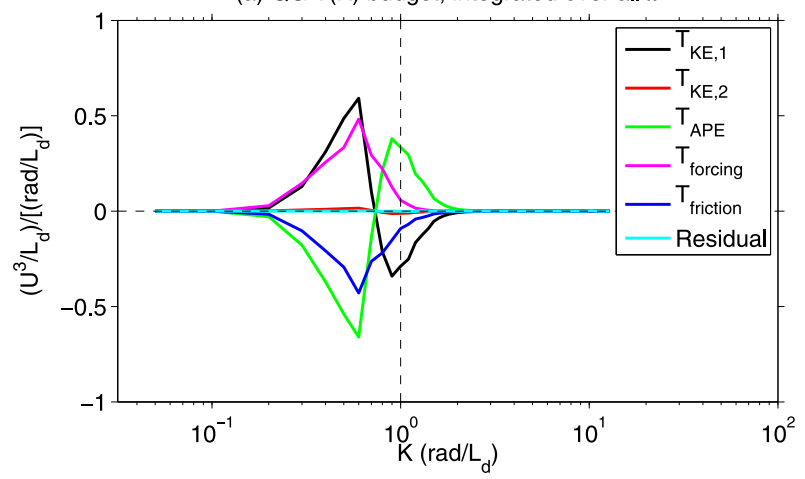

(b) Agulhas $\mathrm{T}_{\mathrm{KE}, 1}(\mathrm{~K})$, integrated over all $\omega$

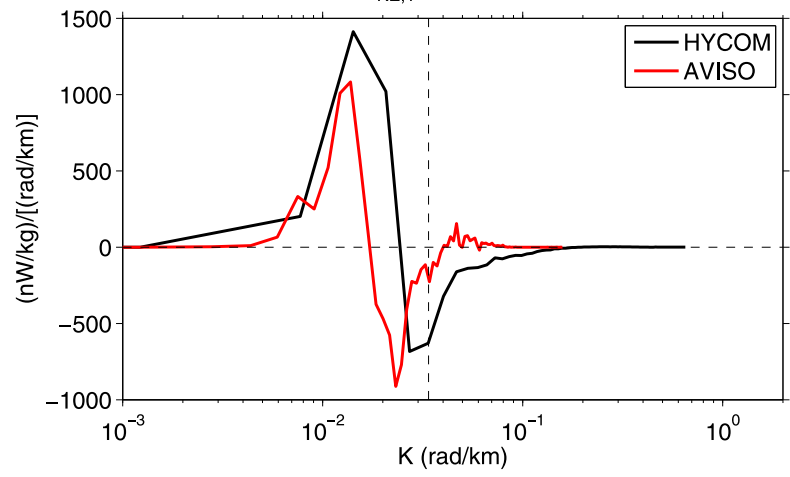

FIG. 13. Wavenumber domain spectral transfers $T(K)$, integrated over all $\omega$. (a) Upper-layer spectral kinetic energy transfers $T_{\mathrm{KE}, 1}(K)$-black curve-in idealized two-layer QG simulation, shown against other terms in the depth-averaged spectral transfer budget [Eq. (12)]. Dashed vertical line represents the deformation wavenumber $1 \mathrm{rad} / L_{d}$. (b) Ocean surface spectral kinetic energy transfers $T_{\mathrm{KE}, 1}(K)$ for Agulhas region HYCOM and AVISO output. Dashed vertical line represents the deformation wavenumber $1 \mathrm{rad} / L_{d}$ for the Agulhas region (see text).

secondary contributors to the spectral transfer $T(K)$ budget. The HYCOM and AVISO wavenumber domain spectral kinetic energy transfers $T_{\mathrm{KE}, 1}(K)$ (Fig. 13b) are similar to those in the QG simulation, in that negative lobes are seen near the $L_{d}$ scale and positive lobes are seen at lower wavenumbers.

Figure 14a shows the frequency domain spectral transfer budget [Eq. (12)] for the QG model. The negative lobe in the upper-layer nonlinear spectral kinetic energy transfer $T_{\mathrm{KE}, 1}(\omega)$-black curve-implies that nonlinearities extract energy near the $L_{d} / U$ time scale associated with baroclinic instability (indicated by vertical dashed line). The nonlinear terms deposit this energy into lower frequencies, as indicated by positive black curve values at lower frequencies. To an even greater extent than in the wavenumber domain transfers $T(K)$, the largest frequency domain transfers $T(\omega)$ at lower frequencies are the nonlinear upper-layer kinetic energy and APE terms, which to the first order balance 
(a) QG T( $\omega)$ budget, integrated over all K

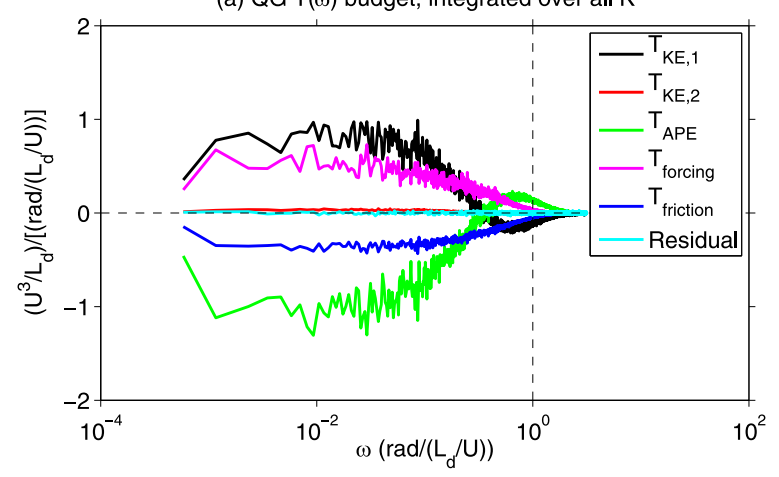

(b) Agulhas $\mathrm{T}_{\mathrm{KE}, 1}(\omega)$, integrated over all $\mathrm{K}$ and logarithmically smoothed

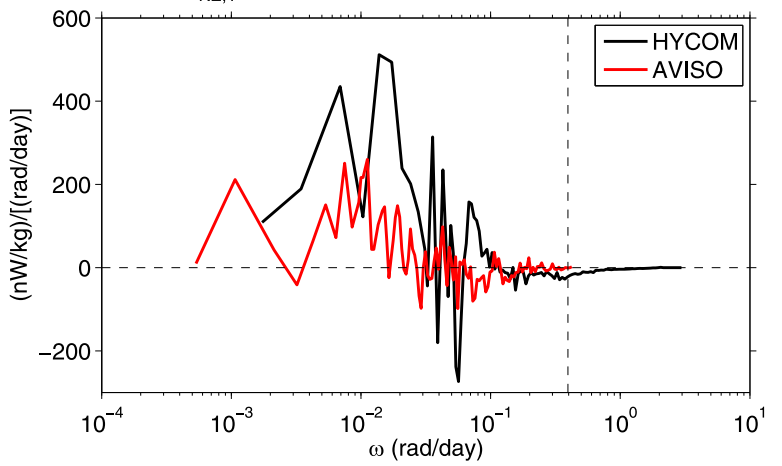

FIG. 14. Frequency domain spectral transfers $T(\omega)$, integrated over all $K$. (a) Upper-layer spectral kinetic energy transfers $T_{\mathrm{KE}, 1}(\omega)$-black curve-in idealized two-layer QG simulation, shown against other terms in the depth-averaged spectral transfer budget [Eq. (12)]. Dashed vertical line represents the frequency $1 \mathrm{rad} /\left(L_{d} / U\right)$, where $U$ is the imposed mean shear $\bar{u}_{1}-\bar{u}_{2}$. (b) Logarithmically smoothed ocean surface spectral kinetic energy transfers $T_{\mathrm{KE}, 1}(\omega)$ for Agulhas region HYCOM and AVISO output. Dashed vertical line represents the frequency $1 \mathrm{rad} /\left(L_{d} / U\right)$, where $L_{d}$ is the deformation radius and $U$ is the estimated mean shear for the Agulhas region (see text).

each other out. The forcing and friction terms are important, but only about half as large as the nonlinear kinetic and potential energy transfers. Thus, at least in our simple QG simulation, nonlinearities are the most important terms in the maintenance of low-frequency energy.

Figure 14b shows Agulhas HYCOM and AVISO $T_{\mathrm{KE}, 1}(\omega)$ curves, which have been logarithmically smoothed here for display purposes (no smoothing of $T$ has been done elsewhere in this paper). At low frequencies the HYCOM and AVISO $T_{\mathrm{KE}, 1}(\omega)$ curves are predominantly positive, as in the QG results, albeit noisy. Equation (20) demonstrates that any $\Pi_{\mathrm{KE}, 1}(\omega)$ curve having predominantly negative values at higher frequencies-such as occurs in the Agulhas region for both HYCOM and AVISO-must be associated with predominantly negative spectral transfer values at high frequencies. A negative lobe can be seen in the HYCOM $T_{\mathrm{KE}, 1}(\omega)$ curve on either side of the baroclinic instability time scale $L_{d} / U$ (vertical dashed line), as in the QG results. The AVISO transfers are predominantly negative at higher frequencies, though noisy, and shifted to lower frequencies relative to HYCOM, as in the spectral flux results.

\section{Summary and discussion}

Following our earlier examination of geostrophic turbulence in the frequency domain (ASFMRS), this paper has examined geostrophic turbulence in the frequency-wavenumber domain. We are motivated by Penduff et al. (2011) and related studies, which point to the potential of oceanic mesoscale eddies to drive significant low-frequency oceanic variability. The frequency-wavenumber diagnostics developed here and in ASFMRS represent a relatively little-known tool for studying low-frequency oceanic variability [to our knowledge, the only previous usage of such diagnostics is in the atmospheric literature, e.g., Sheng and Hayashi (1990a,b)]. Frequency-wavenumber spectra, spectral fluxes, and spectral transfers from an idealized forceddissipated QG turbulence simulation, a realistic eddying HYCOM simulation, and AVISO gridded satellite altimeter maps exhibit several similarities. The range of frequencies that are energetic and participate strongly in nonlinear interactions is greater than the range of energetic and active wavenumbers. Consideration of joint frequency-wavenumber spectral fluxes yields the important constraint that the frequency domain spectral flux at the lowest frequency must be zero for geostrophic flows. Application of this constraint gives HYCOM frequency domain fluxes and transfers that are more robust, and more consistent with QG model behaviors, than the frequency domain spectral fluxes computed from realistic models in ASFMRS. In five of the six regions examined in this paper, the realistic HYCOM simulation exhibits a kinetic energy cascade toward lower frequencies alongside the well-known inverse cascade toward lower wavenumbers, as in the QG model. AVISO spectral fluxes show a cascade to lower frequencies in some regions, but not in others. However, the discrepancies of the AVISO results with QG and HYCOM results are likely due at least in part to the filtering inherent in the creation of the AVISO product. Exercises performed with HYCOM output indicate that such filtering distorts the computed frequency domain spectral fluxes substantially. In the QG simulations, spectral transfer budgets demonstrate that nonlinear transfers of kinetic and potential energy are the primary contributors to the maintenance of low-frequency, lowwavenumber energy, with forcing and friction playing important but secondary roles. Thus, in the QG model at 
least, low-frequency variability is more due to intrinsic nonlinearities than to forcing.

Low-frequency energy is associated with low wavenumbers, and vice versa, though not in a simple way. Spectral slopes in wavenumber and frequency space are not equal, as they would be if Taylor (1938)'s hypothesis, which relates frequency to wavenumber spectra, held precisely. The limitation of the Taylor (1938) hypothesis in our results is consistent with discussions in Hayashi and Golder (1977), who found no simple relationship between the frequency and wavenumber spectra of kinetic energy in realistic atmospheric models and atmospheric datasets.

Acknowledgments. We thank three anonymous reviewers whose comments greatly improved the manuscript. Further helpful discussions with Glenn Flierl, Steve Griffies, Joe Pedlosky, and Carl Wunsch are gratefully acknowledged. BKA and AJM acknowledge support from Office of Naval Research (ONR) Grant N00014-110487, National Science Foundation (NSF) Grant OCE0960820, and University of Michigan faculty startup funds. MM acknowledges support from a subcontract of the ONR grant above to the University of Victoria. JFS and JGR acknowledge support from the projects "Global and remote littoral forcing in global ocean models" and "Ageostrophic vorticity dynamics of the ocean," respectively, both sponsored by ONR under Program Element 601153N. RBS acknowledges support from Centre National de la Recherche Scientifique (CNRS), NSF Grants OCE-0960834 and OCE-0851457, a contract with the National Oceanography Centre, Southampton, and a NASA subcontract to Boston University. GS and TP acknowledge support from CNES. This work is a contribution to the CHAOCEAN project, supported by the CNES/NASA Ocean Surface Topography Science Team (OST/ST). The NRL contribution has been approved for public release.

\section{REFERENCES}

Arbic, B. K., and G. R. Flierl, 2004: Baroclinically unstable geostrophic turbulence in the limits of strong and weak bottom Ekman friction: Application to midocean eddies. J. Phys. Oceanogr., 34, 2257-2273, doi:10.1175/1520-0485(2004)034<2257: BUGTIT $>2.0 . \mathrm{CO} ; 2$.

— A. J. Wallcraft, and E. J. Metzger, 2010: Concurrent simulation of the eddying general circulation and tides in a global ocean model. Ocean Modell., 32, 175-187, doi:10.1016/ j.ocemod.2010.01.007.

_ J. J. Richman, J. F. Shriver, P. G. Timko, E. J. Metzger, and A. J. Wallcraft, 2012a: Global modeling of internal tides within an eddying ocean general circulation model. Oceanography, 25, 20-29, doi:10.5670/oceanog.2012.38.

—, R. B. Scott, G. R. Flierl, A. J. Morten, J. G. Richman, and J. F. Shriver, 2012b: Nonlinear cascades of surface oceanic geostrophic kinetic energy in the frequency domain. J. Phys. Oceanogr., 42, 1577-1600, doi:10.1175/JPO-D-11-0151.1.

- K. L. Polzin, R. B. Scott, J. G. Richman, and J. F. Shriver, 2013: On eddy viscosity, energy cascades, and the horizontal resolution of gridded satellite altimeter products. J. Phys. Oceanogr., 43, 283-300, doi:10.1175/JPO-D-11-0240.1.

Batchelor, G. K., 1953: The Theory of Homogeneous Turbulence. Cambridge University Press, 121 pp.

Berloff, P. S., and J. C. McWilliams, 1999: Large-scale, lowfrequency variability in wind-driven ocean gyres. J. Phys. Oceanogr., 29, 1925-1949, doi:10.1175/1520-0485(1999)029<1925: LSLFVI $>2.0 . \mathrm{CO} ; 2$.

Canuto, C., M. Y. Hussaini, A. Quarteroni, and T. A. Zang, 1988: Spectral Methods in Fluid Mechanics. Springer-Verlag, 568 pp.

Charney, J. G., 1971: Geostrophic turbulence. J. Atmos. Sci., 28, 10871095, doi:10.1175/1520-0469(1971)028<1087:GT>2.0.CO;2.

Chassignet, E. P., H. E. Hurlburt, O. M. Smedstad, G. R. Halliwell, P. J. Hogan, A. J. Wallcraft, R. Baraille, and R. Bleck, 2007: The HYCOM (Hybrid Coordinate Ocean Model) data assimilative system. J. Mar. Syst., 65, 60-83, doi:10.1016/ j.jmarsys.2005.09.016.

Chelton, D. B., and M. G. Schlax, 1996: Global observations of oceanic Rossby waves. Science, 272, 234-238, doi:10.1126/ science.272.5259.234.

_, R. A. deSzoeke, M. G. Schlax, K. El Naggar, and N. Siwertz, 1998: Geographical variability of the first-baroclinic Rossby radius of deformation. J. Phys. Oceanogr., 28, 433-460, doi:10.1175/1520-0485(1998)028<0433:GVOTFB >2.0.CO;2.

—, M. G. Schlax, and R. M. Samelson, 2011: Global observations of nonlinear mesoscale eddies. Prog. Oceanogr., 91, 167-216, doi:10.1016/j.pocean.2011.01.002.

Dewar, W. K., 2001: On ocean dynamics in midlatitude climate. J. Climate, 14, 4380-4397, doi:10.1175/1520-0442(2001)014<4380: OODIMC $>2.0 . \mathrm{CO} ; 2$.

Dijkstra, H. A., and M. J. Molemaker, 1999: Imperfections of the North Atlantic wind-driven ocean circulation: Continental geometry and windstress shape. J. Mar. Res., 57, 1-28, doi:10.1357/002224099765038544.

Ducet, N., P.-Y. Le Traon, and G. Reverdin, 2000: Global highresolution mapping of ocean circulation from TOPEX/Poseidon and ERS-1 and -2. J. Geophys. Res., 105, 19477-19498, doi:10.1029/2000JC900063.

Early, J. J., R. M. Samelson, and D. B. Chelton, 2011: The evolution and propagation of quasigeostrophic ocean eddies. J. Phys. Oceanogr., 41, 1535-1555, doi:10.1175/2011JPO4601.1.

Farrar, J. T., 2008: Observations of the dispersion characteristics and meridional sea level structure of equatorial waves in the Pacific Ocean. J. Phys. Oceanogr., 38, 1669-1689, doi:10.1175/ 2007JPO3890.1.

Ferrari, R., and C. Wunsch, 2010: The distribution of eddy kinetic and potential energies in the global ocean. Tellus, 62A, 92108, doi:10.1111/j.1600-0870.2009.00432.x.

Fjortoft, R., 1953: On the changes in the spectral distribution of kinetic energy for two-dimensional nondivergent flow. Tellus, 5, 225-230, doi:10.1111/j.2153-3490.1953.tb01051.x.

Flierl, G. R., 1978: Models of vertical structure and the calibration of two-layer models. Dyn. Atmos. Oceans, 2, 341-381, doi:10.1016/0377-0265(78)90002-7.

Fu, L.-L., and G. R. Flierl, 1980: Nonlinear energy and enstrophy transfers in a realistically stratified ocean. Dyn. Atmos. Oceans, 4, 219-246, doi:10.1016/0377-0265(80)90029-9.

Griffies, S. M., and E. Tziperman, 1995: A linear thermohaline oscillator driven by stochastic atmospheric forcing. 
J. Climate, 8,2440-2453, doi:10.1175/1520-0442(1995)008<2440: ALTODB $>2.0 . C O ; 2$.

Haidvogel, D. B., and I. M. Held, 1980: Homogeneous quasigeostrophic turbulence driven by a uniform temperature gradient. J. Atmos. Sci., 37, 2644-2660, doi:10.1175/ 1520-0469(1980)037<2644:HQGTDB > 2.0.CO;2.

Hasselmann, K., 1976: Stochastic climate models. Part I. Theory. Tellus, 28, 473-485, doi:10.1111/j.2153-3490.1976.tb00696.x.

Hayashi, Y., 1982: Space-time spectral analysis and its applications to atmospheric waves. J. Meteor. Soc. Japan, 60, 156-171. , and D. G. Golder, 1977: Space-time spectral analysis of mid-latitude disturbances appearing in a GFDL general circulation model. J. Atmos. Sci., 34, 237-262, doi:10.1175/ 1520-0469(1977)034<0237:STSAOM $>2.0 . C O ; 2$.

Hecht, M. W., and H. Hasumi, Eds., 2008: Ocean Modeling in an Eddying Regime. Geophys. Monogr., Vol. 177, Amer. Geophys. Union, $409 \mathrm{pp}$.

Holland, W., 1978: The role of mesoscale eddies in the general circulation of the ocean-Numerical experiments using a winddriven quasi-gesotrophic model. J. Phys. Oceanogr., 8, 363-392, doi:10.1175/1520-0485(1978)008<0363:TROMEI >2.0.CO;2.

Hua, B. L., and D. B. Haidvogel, 1986: Numerical simulations of the vertical structure of quasi-geostrophic turbulence. J. Atmos. Sci., 43, 2923-2936, doi:10.1175/1520-0469(1986)043<2923: NSOTVS $>2.0 . C O ; 2$.

Hurlburt, H. E., and J. D. Thompson, 1980: A numerical study of Loop Current intrusions and eddy shedding. J. Phys. Oceanogr., 10, 1611-1651, doi:10.1175/1520-0485(1980)010<1611: ANSOLC $>2.0 . \mathrm{CO} ; 2$.

Huybers, P., and W. Curry, 2006: Links between annual, Milankovitch and continuum temperature variability. Nature, 441, 329-332, doi:10.1038/nature04745.

Kraichnan, R. H., 1967: Inertial ranges in two-dimensional turbulence. Phys. Fluids, 10, 1417-1423, doi:10.1063/1.1762301.

_- 1971: Inertial-range transfer in two- and three-dimensional turbulence. J. Fluid Mech., 47, 525-535, doi:10.1017/ S0022112071001216.

LaCasce, J. H., 1996: Baroclinic vortices over a sloping bottom. Ph.D. dissertation, Massachusetts Institute of TechnologyWoods Hole Oceanographic Institution Joint Program in Physical Oceanography, $220 \mathrm{pp}$.

Larichev, V. D., and I. M. Held, 1995: Eddy amplitudes and fluxes in a homogeneous model of fully developed baroclinic instability. J. Phys. Oceanogr., 25, 2285-2297, doi:10.1175/ 1520-0485(1995)025<2285:EAAFIA > 2.0.CO;2.

Le Traon, P.-Y., F. Nadal, and N. Ducet, 1998: An improved mapping method of multisatellite altimeter data. J. Atmos. Oceanic Technol., 15, 522-534, doi:10.1175/1520-0426(1998)015<0522: AIMMOM $>2.0 . \mathrm{CO} ; 2$.

Liu, W. T., and X. Xie, 2006: Measuring ocean surface wind from space. Remote Sensing of the Marine Environment: Manual of Remote Sensing, Vol. 6, J. Gower, Ed., American Society for Photogrammetry and Remote Sensing, 149-178.

Maximenko, N., P. Niiler, M.-H. Rio, O. Melnichenko, L. Centurioni, D. Chambers, V. Zlotnicki, and B. Galperin, 2009: Mean dynamic topography of the ocean derived from satellite and drifting buoy data using three different techniques. J. Atmos. Oceanic Technol., 26, 1910-1919, doi:10.1175/ 2009JTECHO672.1.

McWilliams, J. C., 1984: The emergence of isolated coherent vortices in turbulent flow. J. Fluid Mech., 146, 21-43, doi:10.1017/ S0022112084001750.
Metzger, E. J., O. M. Smedstad, P. G. Thoppil, H. E. Hurlburt, D. S. Franklin, G. Peggion, J. F. Shriver, and A. J. Wallcraft, 2010: Validation test report for the Global Ocean Forecast System V3.0-1/12 ${ }^{\circ}$ HYCOM/NCODA: Phase II. NRL Memo. Rep. NRL/MR/7320-10-9236, 76 pp. [Available online at http:// www7320.nrlssc.navy.mil/pubs/2010/metzger1-2010.pdf.]

Niiler, P. P., N. A. Maximenko, and J. C. McWilliams, 2003: Dynamically balanced absolute sea level of the global ocean derived from near-surface velocity observations. Geophys. Res. Lett., 30, 2164, doi:10.1029/2003GL018628.

Penduff, T., M. Juza, B. Barnier, J. Zika, W. K. Dewar, A.-M. Treguier, J.-M. Molines, and N. Audiffren, 2011: Sea level expression of intrinsic and forced ocean variabilities at interannual time scales. J. Climate, 24, 5652-5670, doi:10.1175/ JCLI-D-11-00077.1.

Priestley, M. B., 1981: Spectral Analysis and Time Series. Academic Press, $890 \mathrm{pp}$.

Richman, J. G., C. Wunsch, and N. G. Hogg, 1977: Space and time scales of mesoscale motion in the western North Atlantic. Rev. Geophys. Space Phys., 15, 385-420, doi:10.1029/ RG015i004p00385.

— B. K. Arbic, J. F. Shriver, E. J. Metzger, and A. J. Wallcraft, 2012: Inferring dynamics from the wavenumber spectra of an eddying global ocean model with embedded tides. J. Geophys. Res., 117, C12012, doi:10.1029/2012JC008364.

Rosmond, T. E., J. Teixeira, M. Peng, T. F. Hogan, and R. Pauley, 2002: Navy Operational Global Atmospheric Prediction System (NOGAPS): Forcing for ocean models. Oceanography, 15, 99-108, doi:10.5670/oceanog.2002.40.

Salmon, R., 1978: Two-layer quasi-geostrophic turbulence in a simple special case. Geophys. Astrophys. Fluid Dyn., 10, $25-$ 52, doi:10.1080/03091927808242628.

- 1980: Baroclinic instability and geostrophic turbulence. Geophys. Astrophys. Fluid Dyn., 15, 167-211, doi:10.1080/ 03091928008241178.

Sasaki, H., and P. Klein, 2012: SSH wavenumber spectra in the North Pacific from a high-resolution realistic simulation. J. Phys. Oceanogr., 42, 1233-1241, doi:10.1175/JPO-D-11-0180.1.

Schlösser, F., and C. Eden, 2007: Diagnosing the energy cascade in a model of the North Atlantic. Geophys. Res. Lett., 34, L02604, doi:10.1029/2006GL027813.

Scott, R. B., and F. Wang, 2005: Direct evidence of an oceanic inverse kinetic energy cascade from satellite altimetry. J. Phys. Oceanogr., 35, 1650-1666, doi:10.1175/JPO2771.1.

— turbulence: Implications for ocean energetics. J. Phys. Oceanogr., 37, 673-688, doi:10.1175/JPO3027.1.

Sheng, J., and Y. Hayashi, 1990a: Observed and simulated energy cycles in the frequency domain. J. Atmos. Sci., 47, 1243-1254, doi:10.1175/1520-0469(1990)047<1243:OASECI>2.0.CO;2.

$\longrightarrow$, and $\longrightarrow$, 1990b: Estimation of atmospheric energetics in the frequency domain during the FGGE year. J. Atmos. Sci., 47, 1255-1268, doi:10.1175/1520-0469(1990)047<1255: EOAEIT>2.0.CO;2.

Shriver, J. F., H. E. Hurlburt, O. M. Smedstad, A. J. Wallcraft, and R. C. Rhodes, 2007: $1 / 32^{\circ}$ real-time global ocean prediction and value-added over $1 / 16^{\circ}$ resolution. J. Mar. Syst., 65, 3-26, doi:10.1016/j.jmarsys.2005.11.021.

—, B. K. Arbic, J. G. Richman, R. D. Ray, E. J. Metzger, A. J. Wallcraft, and P. G. Timko, 2012: An evaluation of the barotropic and internal tides in a high-resolution global ocean circulation model. J. Geophys. Res., 117, C10024, doi:10.1029/ 2012JC008170. 
Spall, M., 1996: Dynamics of the Gulf Stream/deep western boundary current crossover. Part II: Low-frequency internal oscillations. J. Phys. Oceanogr., 26, 2169-2182, doi:10.1175/ 1520-0485(1996)026<2169:DOTGSW >2.0.CO;2.

Taguchi, B., B. Qiu, M. Nonaka, H. Sasaki, S.-P. Xie, and N. Schneider, 2010: Decadal variability of the Kuroshio Extension: Mesoscale eddies and recirculations. Ocean Dyn., 60 , 673-691, doi:10.1007/s10236-010-0295-1.

Taylor, G. I., 1938: The spectrum of turbulence. Proc. Roy. Soc. London, 164, 476-490, doi:10.1098/rspa.1938.0032.

Tennekes, H., 1975: Eulerian and Lagrangian time microscales in isotropic turbulence. J. Fluid Mech., 67, 561-567, doi:10.1017/ S0022112075000468.

-, and J. L. Lumley, 1972: A First Course in Turbulence. MIT Press, 300 pp.

Thoppil, P. G., J. G. Richman, and P. J. Hogan, 2011: Energetics of a global ocean circulation model compared to observations. Geophys. Res. Lett., 38, L15607, doi:10.1029/2011GL048347.

Tulloch, R., J. Marshall, C. Hill, and K. S. Smith, 2011: Scales, growth rates, and spectral fluxes of baroclinic instability in the ocean. J. Phys. Oceanogr., 41, 1057-1076, doi:10.1175/ 2011JPO4404.1.

Vallis, G. K., 2006: Atmospheric and Oceanic Fluid Dynamics: Fundamentals and Large-Scale Circulation. Cambridge University Press, $745 \mathrm{pp}$.

Wortham, C. J. L., IV, 2013: A multi-dimensional spectral description of ocean variability with applications. Ph.D. dissertation, Massachusetts Institute of Technology-Woods Hole Oceanographic Institution Joint Program in Physical Oceanography, $184 \mathrm{pp}$.

Wunsch, C., 2009: The oceanic variability spectrum and transport trends. Atmos.-Ocean, 47, 281-291, doi:10.3137/ OC310.2009.

2010: Toward a midlatitude ocean frequency-wavenumber spectral density and trend determination. J. Phys. Oceanogr., 40, 2264-2281, doi:10.1175/2010JPO4376.1.

, and D. Stammer, 1995: The global frequency-wavenumber spectrum of oceanic variability estimated from TOPEX/ POSEIDON altimetric measurements. J. Geophys. Res., 100, 24 895-24 910, doi:10.1029/95JC01783. 